\title{
A classifying space for commutativity in Lie groups
}

\author{
ALEJANDRO ADEM \\ José MANUEL Gómez
}

\begin{abstract}
In this article we consider a space $B_{\mathrm{com}} G$ assembled from commuting elements in a Lie group $G$ first defined by Adem, Cohen and Torres-Giese. We describe homotopy-theoretic properties of these spaces using homotopy colimits, and their role as a classifying space for transitionally commutative bundles. We prove that $\mathbb{Z} \times B_{\text {com }} U$ is a loop space and define a notion of commutative $\mathrm{K}$-theory for bundles over a finite complex $X$, which is isomorphic to $\left[X, \mathbb{Z} \times B_{\text {com }} U\right]$. We compute the rational cohomology of $B_{\text {com }} G$ for $G$ equal to any of the classical groups $\mathrm{SU}(r)$, $U(q)$ and $\operatorname{Sp}(k)$, and exhibit the rational cohomologies of $B_{\mathrm{com}} U, B_{\mathrm{com}} \mathrm{SU}$ and $B_{\text {com }} \mathrm{Sp}$ as explicit polynomial rings.
\end{abstract}

\section{E99; 55R35}

\section{Introduction}

Let $G$ denote a topological group and consider the spaces $\left\{\operatorname{Hom}\left(\mathbb{Z}^{n}, G\right)\right\}_{n \geq 0}$ of ordered commuting $n$-tuples in $G$. Adem, Cohen and Torres-Giese [3] showed that they can be assembled into a simplicial space where the resulting geometric realization, denoted here by $B_{\text {com }} G$, is the first term in an increasing filtration of $B G$. The universal bundle over $B G$ pulls back to a principal bundle over $B_{\text {com }} G$ with total space $E_{\text {com }} G$ that can also be described simplicially (see Section 2). In this paper we analyze the properties of $E_{\text {com }} G$ and $B_{\text {com }} G$ when $G$ is a Lie group. We also study variants of our constructions, denoted $E_{\mathrm{com}} G_{\mathbf{1}}$ and $B_{\mathrm{com}} G_{\mathbf{1}}$, which arise from the components of the identity in the commuting varieties (see Section 3 for details). These two constructions agree if the spaces $\operatorname{Hom}\left(\mathbb{Z}^{n}, G\right)$ are path-connected for every $n \geq 0$. It can be shown that for a compact Lie group $G$, this condition is equivalent to the property that the maximal abelian subgroups of $G$ are precisely the maximal tori (see the proof of Adem and Gómez [4, Proposition 2.5]). For example, this condition holds for the classical groups $\mathrm{SU}(r), U(q)$ and $\mathrm{Sp}(k)$ (see Borel [9, Theorem 5.2]), and therefore for any of their finite cartesian products.

We start by applying the recent work of Pettet and Souto [23] to reduce matters to compact Lie groups: 
Theorem 3.1 If $G$ is a real or complex reductive algebraic group with maximal compact subgroup $K$, then the inclusion map $K \subset G$ induces homotopy equivalences $B_{\mathrm{com}} K \simeq B_{\mathrm{com}} G$ and $E_{\mathrm{com}} K \simeq E_{\mathrm{com}} G$.

A similar statement is true for the variants $E_{\mathrm{com}} G_{\mathbf{1}}$ and $B_{\mathrm{com}} G_{\mathbf{1}}$ (see Section 3 for details). Based on this we can focus on the case of a compact connected Lie group $G$. The connected component of the identity in the commuting variety $\operatorname{Hom}\left(\mathbb{Z}^{n}, G\right)$ has the key feature that any $n$-tuple in it can be conjugated into a maximal torus in $G$. Using this we obtain a natural identification $B_{\text {com }} G_{\mathbf{1}} \cong \operatorname{colim}_{S \in \mathcal{T}(G)} B S$, where $\mathcal{T}(G)$ is the topological poset formed by the maximal tori and their intersections under inclusion (see Definition 5.3). We describe the homotopy of these spaces using more tractable homotopy colimits defined over a discrete category. Let $Z=Z(G)$ be the center of $G$ and write $n=\operatorname{rank}(G)-\operatorname{rank}(Z) \geq 0$. Consider the poset $\mathcal{S}(n)$ consisting of all the nonempty subsets of $\{0,1, \ldots, n\}$, with the order given by the reverse inclusion of sets. For each $G$ we have functors $\mathcal{F}_{G}, \mathcal{H}_{G}: \mathcal{S}(n) \rightarrow$ Top such that the following holds (see Section 6 for the definitions of $\mathcal{F}_{G}$ and $\mathcal{H}_{G}$ ).

Theorem 6.3 Suppose that $G$ is a compact, connected Lie group. Then there is a natural homotopy equivalence hocolim $i \in \mathcal{S}(n) \mathcal{F}_{G}(\boldsymbol{i}) \simeq B_{\text {com }} G_{\mathbf{1}}$.

Theorem 6.5 Suppose that $G$ is a compact connected Lie group. Then there is a natural $G$-equivariant homotopy equivalence $\operatorname{hocolim}_{\boldsymbol{i} \in \mathcal{S}(n)} \mathcal{H}_{G}(\boldsymbol{i}) \simeq E_{\mathrm{com}} G_{\mathbf{1}}$.

In terms of bundle theory, we prove that $B_{\mathrm{com}} G$ is a classifying space for bundles that are transitionally commutative.

Theorem 2.2 Suppose that $G$ is a Lie group and let $f: X \rightarrow B G$ denote the classifying map of a principal $G$-bundle $q: E \rightarrow X$ over the finite $C W$-complex $X$. Then up to homotopy, $f$ factors through $B_{\mathrm{com}} G$ if and only if there is an open cover of $X$ on which the bundle is trivial over each open set and such that on intersections the transition functions commute when they are simultaneously defined.

From this we can define the notion of equivalence between transitionally commutative vector bundles and thus define commutative $\mathrm{K}$-theory $K_{\text {com }}(X)$ in a manner analogous to ordinary complex $\mathrm{K}$-theory. Let $U=\operatorname{colim}_{n \rightarrow \infty} U(n)$; then we can establish that $B_{\text {com }} U$ plays a role similar to $B U$ :

Theorem 4.1 The space $\mathbb{Z} \times B_{\text {com }} U$ is a loop space and for any finite $C W$-complex $X$ there is a natural isomorphism of groups $K_{\text {com }}(X) \cong\left[X, \mathbb{Z} \times B_{\text {com }} U\right]$. 
Adem, Gómez, Lind and Tillmann [5] proved that $\mathbb{Z} \times B_{\text {com }} U$ is in fact an infinite loop space so that commutative $\mathrm{K}$-theory forms part of a generalized cohomology theory. Having established the role played by $B_{\text {com }} G$ in bundle theory, it seems natural to compute its cohomology. As can be seen from the homotopy colimit model and computations for SU(2) (see Example 6.4), we expect these spaces to have rather intricate torsion. Here we focus on calculations for the rational cohomology (inverting the order of the Weyl group $W$ would suffice).

Theorem 7.2 Suppose that $G$ is a compact, connected Lie group. Then

$$
H^{*}\left(B_{\mathrm{com}} G_{\mathbf{1}} ; \mathbb{Q}\right)
$$

is a free module over $H^{*}(B G ; \mathbb{Q})$ of rank $|W|$, where $W$ is the corresponding Weyl group.

On the other hand, by [3, Theorem 6.1] we have a natural isomorphism

$$
H^{*}\left(B_{\mathrm{com}} G_{\mathbf{1}} ; \mathbb{Q}\right) \cong\left(H^{*}(G / T ; \mathbb{Q}) \otimes H^{*}(B T ; \mathbb{Q})\right)^{W},
$$

where the Weyl group $W$ acts diagonally on the tensor product. As a corollary we deduce the following algebra isomorphism:

Corollary 7.4 Suppose that $G$ is a connected compact Lie group with maximal torus $T$ and associated Weyl group $W$. Then there is a natural isomorphism of rings

$$
H^{*}\left(E_{\mathrm{com}} G_{\mathbf{1}}\right) \cong\left(H^{*}(G / T) \otimes H^{*}(G / T)\right)^{W},
$$

and the Poincaré series of $B_{\mathrm{com}} G_{1}$ and $E_{\mathrm{com}} G_{1}$ satisfy

$$
P_{B_{\mathrm{com}} G_{\mathbf{1}}}(t)=P_{B G}(t) P_{E_{\mathrm{com}} G_{\mathbf{1}}}(t) .
$$

From this we derive the following.

Corollary 7.5 These statements are equivalent for a compact connected Lie group $G$ :

(1) $E_{\text {com }} G_{1}$ is contractible.

(2) $E_{\mathrm{com}} G_{\mathbf{1}}$ is rationally acyclic.

(3) $G$ is abelian.

Using the theory of multisymmetric polynomials in Section 8 we provide combinatorial descriptions and Poincare series for these algebras in the case of the classical groups $\mathrm{SU}(r), U(q)$ and $\mathrm{Sp}(k)$. Taking limits we obtain that the algebras $H^{*}\left(B_{\mathrm{com}} U ; \mathbb{Q}\right)$, $H^{*}\left(B_{\text {com }} \mathrm{SU} ; \mathbb{Q}\right)$ and $H^{*}\left(B_{\text {com }} \mathrm{Sp} ; \mathbb{Q}\right)$ are polynomial algebras on countably many 
generators (Corollaries 8.3, 8.5 and 8.9, respectively). For example we have an isomorphism of $\mathbb{Q}$-algebras

$$
H^{*}\left(B_{\mathrm{com}} U ; \mathbb{Q}\right) \cong \mathbb{Q}\left[z_{a, b} \mid(a, b) \in \mathbb{N}^{2} \text { and } b>0\right],
$$

where the elements $z_{a, b}$ are polynomial generators of degree $2 a+2 b$.

This paper is organized as follows. In Section 2 we describe basic properties of the spaces $E_{\text {com }} G$ and $B_{\text {com }} G$ for $G$ a topological group. In Section 3 we focus on the case when $G$ is a Lie group. In Section 4 we introduce commutative $\mathrm{K}$-theory. Section 5 describes the topological poset generated by the maximal tori in a Lie group $\mathcal{T}(G)$. In section Section 6 we derive the decompositions of $B_{\text {com }} G_{\mathbf{1}}$ and $E_{\text {com }} G_{1}$ as homotopy colimits. Section 7 deals with cohomology calculations. In Section 8 we consider the particular cases when $G=\mathrm{SU}(n), U(n)$ and $\mathrm{Sp}(n)$ and finally in the appendix it is proved that $\left[B_{\mathrm{com}} G\right]_{*}$ is a proper simplicial space for any Lie group $G$. We are grateful to the referee for providing helpful comments.

Acknowledgements The first author was supported by NSERC. The second author would like to thank PIMS for hosting him when part of this work was completed.

\section{Definitions and basic properties of the spaces $B_{\text {com }} G$ and $E_{\text {com }} G$}

In this section we study general properties of the spaces $B_{\text {com }} G$ and $E_{\text {com }} G$, which are constructed by assembling the different spaces of ordered commuting $k$-tuples in a topological group $G$. These spaces were first introduced in [3] where their basic properties were derived, mostly for the case of finite groups.

Suppose that $G$ is a topological group. For technical reasons we will assume that $G$ is locally compact, Hausdorff and that $1_{G} \in G$ is a nondegenerate basepoint. We can associate to $G$ a simplicial space, denoted by $\left[B_{\mathrm{com}} G\right]_{*}$, in the following way. For any integer $n \geq 0$ define

$$
\left[B_{\mathrm{com}} G\right]_{n}:=\operatorname{Hom}\left(\mathbb{Z}^{n}, G\right) \subset G^{n} .
$$

Note that $\left[B_{\text {com }} G\right]_{n}$ can be identified with the subset of $G^{n}$ consisting of all ordered commuting $n$-tuples in the group $G$, and as such it is given the subspace topology. The face and degeneracy maps are defined by

$$
\begin{aligned}
& s_{j}\left(g_{1}, \ldots, g_{n}\right)=\left(g_{1}, \ldots, g_{j}, 1_{G}, g_{j+1}, \ldots, g_{n}\right), \\
& \partial_{i}\left(g_{1}, \ldots, g_{n}\right)= \begin{cases}\left(g_{2}, \ldots, g_{n}\right) & \text { if } i=0, \\
\left(g_{1}, \ldots, g_{i} g_{i+1}, \ldots, g_{n}\right) & \text { if } 0<i<n, \\
\left(g_{1}, \ldots, g_{n-1}\right) & \text { if } i=n .\end{cases}
\end{aligned}
$$


The different $s_{i}$ and $\partial_{j}$ are well-defined and satisfy the simplicial identities, as these maps are precisely the restrictions of the degeneracy and face maps in the bar construction $[B G]_{*}$. We denote by $B_{\text {com }} G$ the geometric realization of the simplicial space $\left[B_{\mathrm{com}} G\right]_{*}$. As shown in [3], the space $B_{\mathrm{com}} G$ is in fact the first space in an increasing filtration of the classifying space of $G$ defined using the descending central series of the free groups. Similarly we can define $\left[E_{\mathrm{com}} G\right]_{n}:=\operatorname{Hom}\left(\mathbb{Z}^{n}, G\right) \times G \subset G^{n+1}$ and use the analogous face and degeneracy maps to define a simplicial space $\left[E_{\mathrm{com}} G\right]_{*}$ and its geometric realization $E_{\mathrm{com}} G$.

The projection on the first $n$-coordinates $\left[E_{\mathrm{com}} G\right]_{*} \rightarrow\left[B_{\mathrm{com}} G\right]_{*}$ defines a simplicial map and therefore at the level of geometric realizations we obtain a continuous map $p_{\text {com }}: E_{\text {com }} G \rightarrow B_{\text {com }} G$. This defines a principal $G$-bundle that can be seen as the restriction of the universal principal $G$-bundle $p: E G \rightarrow B G$, and we have a morphism of principal $G$-bundles that fits into the following diagram:

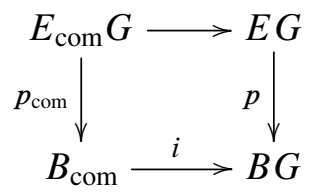

Note that up to homotopy this gives rise to a fibration sequence $E_{\text {com }} G \rightarrow B_{\text {com }} G \rightarrow B G$. Recall that the bundle $p: E G \rightarrow B G$ is universal in the sense that if $q: E \rightarrow X$ is a principal $G$-bundle over a CW-complex $X$, then we can find a continuous map $f: X \rightarrow B G$ such that $q: E \rightarrow X$ is isomorphic to $f^{*} p: f^{*}(E G) \rightarrow X$.

Definition 2.1 Suppose that $X$ is a CW-complex. We say that a principal $G$-bundle $q: E \rightarrow X$ is transitionally commutative if and only if we can find an open cover $\left\{U_{i}\right\}_{i \in I}$ of $X$ such that the bundle $q: E \rightarrow X$ is trivial over each $U_{i}$ and the transition functions $\rho_{i, j}: U_{i} \cap U_{j} \rightarrow G$ commute with each other whenever they are simultaneously defined.

When $G$ is a Lie group the bundle $p_{\mathrm{com}}: E_{\mathrm{com}} G \rightarrow B_{\mathrm{com}} G$ is a universal bundle for transitionally commutative principal $G$-bundles. To make this precise we need to establish the following notation. Let $k \geq 0$ be an integer and consider the standard $k$-simplex

$$
\Delta_{k}=\left\{\left(t_{0}, \ldots, t_{k}\right) \in \mathbb{R}^{k+1} \mid t_{i} \geq 0, \sum_{j=0}^{k} t_{j}=1\right\} .
$$

Note that the symmetric group $\Sigma_{k+1}$ acts by permutation on the vertices of $\Delta_{k}$ and this action can be extended to a linear action on $\Delta_{k}$. Suppose that we have a sequence of integers $\boldsymbol{i}:=\left\{0 \leq i_{1}<\cdots<i_{q} \leq k\right\}$ and let $e_{\boldsymbol{i}}$ denote the element in $\Delta_{k}$ given by $e_{i}=\delta_{i_{q}} \cdots \delta_{i_{1}}\left(\frac{1}{k-q+1}, \ldots, \frac{1}{k-q+1}\right)$, where $\delta_{i_{1}}, \ldots, \delta_{i_{q}}$ denote the different face 
maps. That is, the element $e_{i} \in \Delta_{k}$ has barycentric coordinates $\left(t_{0}, \ldots, t_{k}\right)$ given by

$$
t_{j}= \begin{cases}0 & \text { if } j \in\left\{i_{1}, \ldots, i_{q}\right\}, \\ \frac{1}{k-q+1} & \text { if } \notin\left\{i_{1}, \ldots, i_{q}\right\} .\end{cases}
$$

The length of such a sequence $\boldsymbol{i}$ is defined to be the number $|\boldsymbol{i}|=q$. Observe that if $\boldsymbol{i}$ runs through all the different sequences of integers $\boldsymbol{i}:=\left\{0 \leq i_{1}<\cdots<i_{q} \leq k\right\}$ then the collection $\left\{e_{i}\right\}_{i}$ is precisely the collection of barycenters associated to all the nonempty faces in the standard simplex in $\Delta_{k}$. Let $\left\{W_{q}^{k}\right\}_{q=0}^{k}$ be a collection of open sets in $\Delta_{k}$ satisfying the following properties:

(1) For each $0 \leq q \leq k$ and each $i=\left\{0 \leq i_{1}, \ldots, i_{q} \leq k\right\}$ there is an open neighborhood $V_{\boldsymbol{i}}^{k}$ of $e_{\boldsymbol{i}}$ such that $W_{q}^{k}=\bigsqcup_{|\boldsymbol{i}|=q+1} V_{\boldsymbol{i}}^{k}$.

(2) If $t=\left(t_{0}, \ldots, t_{k}\right) \in V_{\boldsymbol{i}}^{k}$ for some $\boldsymbol{i}=\left\{0 \leq i_{1}<\cdots<i_{q} \leq k\right\}$, then $t_{j}>0$ if $j \notin\left\{i_{1}, \ldots, i_{q}\right\}$.

(3) Each open set $W_{q}^{k}$ is invariant under the action of $\Sigma_{k+1}$.

(4) The sets $\left\{W_{q}^{k}\right\}_{q=0}^{k}$ form an open cover of $\Delta_{k}$.

Clearly such an open cover exists and can be constructed in an inductive way. Using this we have the following geometric description for the bundle $p_{\mathrm{com}}: E_{\mathrm{com}} G \rightarrow B_{\mathrm{com}} G$.

Theorem 2.2 Suppose that $G$ is a Lie group and let $f: X \rightarrow B G$ denote the classifying of a principal $G$-bundle $q: E \rightarrow X$ over the finite $C W$-complex $X$. Then up to homotopy, $f$ factors through $B_{\mathrm{com}} G$ if and only if $q$ is transitionally commutative.

Proof Let $X$ be a finite CW-complex. Assume that the classifying map of $q: E \rightarrow X$ factors through $B_{\text {com }} G$; that is, suppose that the classifying map of this bundle is of the form $f: X \rightarrow B_{\text {com }} G \subset B G$. We will show first that $q$ is transitionally commutative. By Proposition A.1 in the appendix we have that $\left[B_{\mathrm{com}} G\right]_{*}$ is a proper simplicial space (see the appendix for the definition of a proper simplicial space). It follows that the geometric realization of $\left[B_{\mathrm{com}} G\right]_{*}$ is equivalent to Segal's fat geometric realization where the equivalences associated to the degeneracy maps are ignored (see [25, Appendix A]). This realization is denoted here by $\mathbb{B}_{\text {com }} G$, and similarly we have $\mathbb{E}_{\text {com }} G$. For the first part of the proof it will be more convenient for us to work with the (equivalent) principal $G$-bundle $p_{\text {com }}: \mathbb{E}_{\text {com }} G \rightarrow \mathbb{B}_{\text {com }} G$.

By definition

$$
\mathbb{B}_{\mathrm{com}} G:=\left(\bigsqcup_{n \geq 0} \operatorname{Hom}\left(\mathbb{Z}^{n}, G\right) \times \Delta_{n}\right) / \sim,
$$

where $\left(\left(g_{1}, \ldots, g_{k}\right), \delta_{i} u\right) \sim\left(\partial_{i}\left(g_{1}, \ldots, g_{k}\right), u\right)$ with $u \in \Delta_{k-1}$. For each $k \geq 0$, let 


$$
F_{k} \mathbb{B}_{\mathrm{com}} G=\operatorname{Im}\left\{\bigsqcup_{0 \leq n \leq k} \operatorname{Hom}\left(\mathbb{Z}^{n}, G\right) \times \Delta_{n}\right\} \subset \mathbb{B}_{\mathrm{com}} G .
$$

In this way we obtain an increasing filtration of $\mathbb{B}_{\mathrm{com}} G$

$$
F_{0} \mathbb{B}_{\text {com }} G \subset F_{1} \mathbb{B}_{\text {com }} G \subset \cdots \subset F_{k} \mathbb{B}_{\text {com }} G \subset \cdots \subset \mathbb{B}_{\text {com }} G
$$

and $\mathbb{B}_{\text {com }} G=\operatorname{colim}_{k \rightarrow \infty} F_{k} \mathbb{B}_{\text {com }} G$. Since $X$ is a finite CW-complex, we can find some $k \geq 0$ such that the map $f$ factors through $F_{k} \mathbb{B}_{\text {com }} G$; that is, $f: X \rightarrow F_{k} \mathbb{B}_{\text {com }} G \subset$ $\mathbb{B}_{\text {com }} G$. It suffices to show the result for the restriction of the universal principal $G$-bundle $p_{\text {com }}: \mathbb{E}_{\text {com }} G \rightarrow \mathbb{B}_{\text {com }} G$ over $F_{k} \mathbb{B}_{\text {com }} G$ for each $k \geq 0$ fixed. For this fix $\boldsymbol{i}:=\left\{0 \leq i_{1}<\cdots<i_{q} \leq k\right\}$ a sequence of integers and assume that $\left(g_{1}, \ldots, g_{k}\right) \in$ $\operatorname{Hom}\left(\mathbb{Z}^{k}, G\right)=\left[B_{\text {com }} G\right]_{k}$. Then we can see $\left(g_{1}, \ldots, g_{k}, 1\right) \in \operatorname{Hom}\left(\mathbb{Z}^{k}, G\right) \times G=$ $\left[E_{\mathrm{com}} G\right]_{k}$ and we define

$$
\varphi_{\boldsymbol{i}}\left(g_{1}, \ldots, g_{k}\right):=\pi_{k-q}\left(\partial_{i_{1}} \cdots \partial_{i_{q}}\left(g_{1}, \ldots, g_{k}, 1\right)\right)^{-1} .
$$

In this equation, $\partial_{i_{1}}, \ldots, \partial_{i_{q}}$ denote the face maps in the simplicial space $\left[E_{\mathrm{com}} G\right]_{*}$ and $\pi_{n-k}: \operatorname{Hom}\left(\mathbb{Z}^{n-k-1}\right) \times G \rightarrow G$ is the projection onto the last coordinate. For example if $\left(g_{1}, g_{2}, g_{3}\right) \in \operatorname{Hom}\left(\mathbb{Z}^{3}, G\right)$ and $\boldsymbol{i}=\{2,3\}$, then $\varphi_{\boldsymbol{i}}\left(g_{1}, g_{2}, g_{3}\right)=g_{3}^{-1} g_{2}^{-1}$ and if $\boldsymbol{j}=\{1,3\}$ then $\varphi_{\boldsymbol{i}}\left(g_{1}, g_{2}, g_{3}\right)=g_{3}^{-1}$. For any $\left(g_{1}, \ldots, g_{k}\right) \in \operatorname{Hom}\left(\mathbb{Z}^{k}, G\right)$ and any sequence $i$ we have $\varphi_{i}\left(g_{1}, \ldots, g_{k}\right)=g_{j_{1}}^{-1} \cdots g_{j_{r}}^{-1}$ for suitable integers $j_{1}, \ldots, j_{r}$. The functions $\varphi_{i}$ can be used to define local sections of the restriction of the bundle $p_{\text {com }}: \mathbb{E}_{\text {com }} G \rightarrow \mathbb{B}_{\text {com }} G$ over $F_{k} \mathbb{B}_{\text {com }} G$. Indeed, for each $0 \leq q \leq k$ let $U_{q}^{k}$ be the image of $\operatorname{Hom}\left(\mathbb{Z}^{k}, G\right) \times W_{q}^{k}$ in $F_{k} \mathbb{B}_{\text {com }} G$. Thus defined, each $U_{q}^{k}$ is an open set in $F_{k} \mathbb{B}_{\mathrm{com}} G$ and the collection $\left\{U_{q}^{k}\right\}_{q=0}^{k}$ forms an open cover of $F_{k} \mathbb{B}_{\text {com }} G$. Define $\sigma_{q}: U_{q}^{k} \rightarrow p_{\text {com }}^{-1}\left(U_{q}^{k}\right)$ in the following way. Let $x \in U_{q}^{k}$ and write $x=\left[\left(g_{1}, \ldots, g_{k}\right), t\right]$ for some $\left(g_{1}, \ldots, g_{k}\right) \in \Delta_{k}$ and $t \in W_{q}^{k}$. We define $\sigma_{q}(x):=\left[\left(g_{1}, \ldots, g_{k}, \varphi_{i}\left(g_{1}, \ldots, g_{k}\right)\right), t\right]$, provided that $t \in V_{i}^{k}$. The functions $\varphi_{\boldsymbol{i}}$ are defined so that the function $\sigma_{q}$ is well defined and continuous over $U_{q}^{k}$. Thus $\sigma_{q}$ is a continuous section of the restriction of $p_{\text {com }}$ over $U_{q}^{k}$ making it a trivial principal $G$-bundle. With the trivializations provided by these sections, if $x=\left[\left(g_{1}, \ldots, g_{k}\right), t\right]$ in $U_{r}^{k} \cap U_{q}^{k}$, then the transition function $\rho_{r, q}: U_{r}^{k} \cap U_{q}^{k} \rightarrow G$ is such that

$$
x=\left[\left(g_{1}, \ldots, g_{k}\right), t\right] \mapsto g_{j_{0}}^{ \pm 1} \cdots g_{j_{r}}^{ \pm 1}
$$

for a suitable sequence of integers $j_{0}, \ldots, j_{r}$. In particular it follows that the different transition functions $\rho_{r, q}$ are pairwise commutative whenever they are simultaneously defined as $\left(g_{1}, \ldots, g_{k}\right) \in \operatorname{Hom}\left(\mathbb{Z}^{k}, G\right)$ for any $x=\left[\left(g_{1}, \ldots, g_{k}\right), t\right] \in U_{r}^{k} \cap U_{s}^{k}$.

Conversely, suppose that $q: E \rightarrow X$ is a transitionally commutative principal $G$-bundle. Then we can find an open cover $\mathcal{U}:=\left\{U_{i}\right\}_{i \in I}$ of $X$ such that the bundle $q: E \rightarrow X$ is trivial over each $U_{i}$ and the transition functions $\rho_{i, j}: U_{i} \cap U_{j} \rightarrow G$ commute with 
each other whenever they are simultaneously defined. By passing to a refinement of $\mathcal{U}$, we can assume that each nonempty intersection of the sets $U_{i}$ is contractible. Moreover, this cover can be reduced to a countable cover. Let $\mathcal{U}=\left\{U_{i}\right\}_{i \geq 0}$ be the resulting open cover of $X$. For each $i \geq 0$, fix $\varphi_{i}: q^{-1}\left(U_{i}\right) \rightarrow U_{i} \times G$ a trivialization of the restriction of $q$ over $U_{i}$. These trivializations define transition functions $\rho_{i, j}: U_{i} \cap U_{j} \rightarrow G$ whenever $U_{i} \cap U_{j} \neq \varnothing$ and satisfy the cocycle condition $\rho_{i k}=\rho_{i j} \rho_{j k}$ whenever they are defined and are pairwise commutative by assumption. Consider the nerve $N_{*}(\mathcal{U})$ of the cover $\mathcal{U}$. This is a simplicial set with $N_{k}(\mathcal{U})=\bigsqcup_{0 \leq i_{1} \leq \cdots \leq i_{k+1}} U_{i_{1}} \cap \cdots \cap U_{i_{k+1}}$. The different transition functions can be used to define a map of simplicial spaces $\rho_{*}: N_{*}(\mathcal{U}) \rightarrow\left[B_{\mathrm{com}} G\right]_{*}$ in the following way. Suppose that $x \in U_{i_{1}} \cap \cdots \cap U_{i_{k+1}}$ for some $0 \leq i_{1} \leq \cdots \leq i_{k+1}$. Define $\rho_{k}(x)=\left(\rho_{i_{1} i_{2}}(x), \rho_{i_{2} i_{3}}(x), \ldots, \rho_{i_{k} i_{k+1}}(x)\right) \in$ $\operatorname{Hom}\left(\mathbb{Z}^{k}, G\right)$. It is easy to see that this defines a map of simplicial spaces and in particular it induces a continuous map $g=\left|\rho_{*}\right|: N(\mathcal{U}) \rightarrow B_{\text {com }} G$. Since the cover $\mathcal{U}$ was chosen so that each nonempty intersection of sets in $\mathcal{U}$ is contractible, then the natural map $\alpha: N(\mathcal{U}) \rightarrow X$ is a homotopy equivalence (see for example [14, Corollary 4G.3]). Let $\beta: X \rightarrow N(U)$ be a homotopy inverse of $\alpha$. Then $f:=g \circ \beta: X \rightarrow B_{\text {com }} G$ is a continuous map that classifies the principal $G$-bundle $q: E \rightarrow X$.

As a consequence of the proof of the previous theorem, we have that the restriction of the bundle $p_{\text {com }}: \mathbb{E}_{\text {com }} G \rightarrow \mathbb{B}_{\text {com }} G$ to each $F_{k} \mathbb{B}_{\text {com }} G$ defines a transitionally commutative principal $G$-bundle. From this we infer that the bundle $p_{\text {com }}: \mathbb{E}_{\mathrm{com}} G \rightarrow \mathbb{B}_{\mathrm{com}} G$ is itself transitionally commutative, as is the equivalent bundle $p_{\mathrm{com}}: E_{\mathrm{com}} G \rightarrow B_{\mathrm{com}} G$.

As an application of the previous theorem suppose that $G$ is a Lie group and that $X$ is a finite CW-complex for which we can find an open cover $X=U \cup V$ with both $U$ and $V$ contractible. Let $q: E \rightarrow X$ be any principal $G$-bundle over $X$. Then the restriction of $q$ over $U$ and $V$ is trivial since $U$ and $V$ are contractible. Over this trivialization there is only one transition function and thus any such principal $G$-bundle over $X$ is transitionally commutative. By the previous theorem we conclude that the classifying map of the bundle $q: E \rightarrow X$ factors through $B_{\text {com }} G$ up to homotopy. This situation applies in particular to $X=\mathbb{S}^{n}$ for any $n \geq 0$. Therefore the inclusion map $i: B_{\text {com }} G \hookrightarrow B G$ induces a surjective map $i_{\#}:\left[\mathbb{S}^{n}, B_{\text {com }} G\right] \rightarrow\left[\mathbb{S}^{n}, B G\right]$. The following corollary is an immediate consequence after modifying for basepoints and using the fibration $E_{\mathrm{com}} G \rightarrow B_{\mathrm{com}} G \rightarrow B G$.

Corollary 2.3 Let $G$ be a Lie group. Then the map $i: B_{\text {com }} G \rightarrow B G$ induces a surjection $i_{*}: \pi_{n}\left(B_{\mathrm{com}} G\right) \rightarrow \pi_{n}(B G)$ for every $n \geq 0$ and in particular, for every $n \geq 0$ we have a short exact sequence

$$
1 \rightarrow \pi_{n}\left(E_{\mathrm{com}} G\right) \rightarrow \pi_{n}\left(B_{\mathrm{com}} G\right) \stackrel{i_{*}}{\rightarrow} \pi_{n}(B G) \rightarrow 1 .
$$


Remark 2.4 Suppose that $G$ is a connected Lie group. Then by [3, Theorem 6.3] the fibration sequence $\Omega E_{\mathrm{com}} G \rightarrow \Omega B_{\mathrm{com}} G \rightarrow \Omega B G$ has a natural continuous section $\sigma(G): \Omega B G \rightarrow \Omega B_{\text {com }} G$. This implies that for such groups and every $n \geq 0$ the short exact sequence of homotopy groups obtained in the previous corollary splits naturally. Moreover, by [3, Theorem 6.3] there is a natural homotopy equivalence $\theta(G): G \times \Omega E_{\mathrm{com}} G \rightarrow \Omega B_{\mathrm{com}} G$.

Suppose that $X$ is a finite CW-complex. Note that two principal $G$-bundles $q_{0}: E_{0} \rightarrow$ $X$ and $q_{1}: E_{1} \rightarrow X$ are isomorphic if and only if we can find a principal $G$-bundle $p: E \rightarrow X \times[0,1]$ such that $q_{0}=p_{\mid p^{-1}(X \times\{0\})}$ and $q_{1}=p_{\mid p^{-1}(X \times\{1\})}$. Suppose now that $q_{0}: E_{0} \rightarrow X$ and $q_{1}: E_{1} \rightarrow X$ are two transitionally commutative principal $G$-bundles. Then we say that these bundles are transitionally commutative isomorphic if we can find a transitionally commutative principal $G$-bundle $p: E \rightarrow X \times[0,1]$ such that $q_{0}=p_{\mid p^{-1}(X \times\{0\})}$ and $q_{1}=p_{\mid p^{-1}(X \times\{1\})}$. Thus we can identify the set $\left[X, B_{\text {com }} G\right]$ with the set of transitionally commutative isomorphism classes of transitionally commutative principal $G$-bundles over $X$. In other words, the space $B_{\text {com }} G$ is a classifying space for transitionally commutative bundles. If two transitionally commutative principal $G$-bundles are transitionally commutative isomorphic then they are isomorphic as principal $G$-bundles. However, the converse is not true as is demonstrated in the next example.

Example 2.5 Let $G=\mathrm{SU}(2)$ and $T \subset G$ the maximal torus, which in this case is a circle. The quotient $G / T$ can be identified with the sphere $\mathbb{S}^{2}$, let $f: \mathbb{S}^{2} \rightarrow G / T$ be a fixed homeomorphism. Now the action map $G \times T^{n} \rightarrow \operatorname{Hom}\left(\mathbb{Z}^{n}, G\right)$ defined by $\left(g, t_{1}, \ldots, t_{n}\right) \mapsto\left(g t_{1} g^{-1}, \ldots, g t_{n} g^{-1}\right)$ factors through $G / T \times T^{n}$. Looking at the realizations of the respective simplicial spaces, this defines a map $\theta: G / T \times B T \rightarrow$ $B_{\text {com }} G$. According to [3, Theorem 6.1], this gives rise to a rational cohomology isomorphism $H^{*}\left(B_{\text {com }} G, \mathbb{Q}\right) \rightarrow H^{*}(G / T \times B T, \mathbb{Q})^{W}$, where $W=\mathbb{Z} / 2 \mathbb{Z}$ is the Weyl group. This group acts through the sign representation both on the generator $a \in H^{2}(B T, \mathbb{Q})$ and on the top class $b \in H^{2}(G / T, \mathbb{Q})$. The invariant classes $a^{2}$ and $a b$ correspond to a basis for $H^{4}\left(B_{\text {com }} G, \mathbb{Q}\right)$. Now let $g: \mathbb{S}^{2} \rightarrow B T \cong \mathbb{C P}^{\infty}$ be a representative of a generator of $\pi_{2}\left(\mathbb{C P}{ }^{\infty}\right) \cong \mathbb{Z}$. Consider the map $h: \mathbb{S}^{2} \times \mathbb{S}^{2} \rightarrow$ $B_{\text {com }} \mathrm{SU}(2)$ given by the composition $\theta \circ(f \times g)$. In the next section we shall see that $B_{\text {com }} G$ is 3-connected for $G=\mathrm{SU}(2)$, which implies that this map is nullhomotopic on $\mathbb{S}^{2} \vee \mathbb{S}^{2}$ and so defines a map $\tilde{h}: \mathbb{S}^{4} \rightarrow B_{\text {com }} G$. By construction this map is nontrivial in rational cohomology, corresponding to the element $a b$. Moreover, if $i: B_{\text {com }} G \rightarrow B G$ denotes the inclusion map, then the composition $i \circ \tilde{h}$ is trivial in cohomology (as the Chern class in dimension four corresponds to $b^{2}$ ) and so is nullhomotopic. It follows that the principal $G$-bundle over $\mathbb{S}^{4}$ induced by $\tilde{h}$ is trivial as a principal $G$-bundle but not as a transitionally commutative principal $G$-bundle. 
Remark 2.6 If $G=U(k)$ with $k>1$, then the map $i_{*}: \pi_{n}\left(B_{\text {com }} U(k)\right) \rightarrow \pi_{n}(B U(k))$ cannot be an isomorphism for every $n \geq 0$. If this were true $i$ would be a homotopy equivalence; however it follows from [3, Theorem 6.1] that the rational cohomology of $B_{\text {com }} U(k)$ is not isomorphic to that of $B U(k)$. More generally we shall see that if $G$ is a compact connected Lie group which is not abelian, then $E_{\text {com }} G$ is not contractible, unlike the classical universal space $E G$.

\section{Properties of $B_{\mathrm{com}} G$ and $E_{\mathrm{com}} G$ when $G$ is a Lie group}

In this section we focus our attention on the case when $G$ is a real or complex reductive algebraic group. We can consider $G$ as a real or complex Lie group, respectively. Let $K \subset G$ be a maximal compact subgroup; it is well known that such a group always exists and the inclusion map $i: K \hookrightarrow G$ is a strong deformation retract. However, in general there is no retraction $r: G \rightarrow K$ that preserves commutativity; see for example [26] where the nonexistence of such a retraction was proved for the groups $\mathrm{SL}_{n}(\mathbb{C})$ with $n \geq 8$. On the other hand, by [23, Corollary 1.2] the inclusion $\operatorname{Hom}\left(\mathbb{Z}^{n}, K\right) \hookrightarrow$ $\operatorname{Hom}\left(\mathbb{Z}^{n}, G\right)$ is a strong deformation retract. We show here that this can be used to prove that the inclusion $i: B_{\mathrm{com}} K \hookrightarrow B_{\mathrm{com}} G$ is also a strong deformation retract.

Theorem 3.1 Suppose that $G$ is a real or complex reductive algebraic group and let $K$ be a maximal compact subgroup. Then the inclusion map $i: K \hookrightarrow G$ induces homotopy equivalences $i: B_{\mathrm{com}} K \rightarrow B_{\mathrm{com}} G$ and $i: E_{\mathrm{com}} K \rightarrow E_{\mathrm{com}} G$.

Proof We can view $G$ as a (real or complex) Lie group and thus by Proposition A.1 in the appendix $\left[B_{\mathrm{com}} G\right]_{*}$ and $\left[B_{\mathrm{com}} K\right]_{*}$ are proper simplicial spaces. The inclusion map $i: K \hookrightarrow G$ induces a map of simplicial spaces $i_{*}:\left[B_{\mathrm{com}} K\right]_{*} \rightarrow\left[B_{\mathrm{com}} G\right]_{*}$ that is a level-wise homotopy equivalence. By [19, Theorem A.4] we conclude that the induced map at the level of geometric realizations $i: B_{\mathrm{com}} K \rightarrow B_{\mathrm{com}} G$ is a homotopy equivalence. Next we prove that $i: E_{\mathrm{com}} K \rightarrow E_{\mathrm{com}} G$ is a homotopy equivalence. For this, note that the inclusion map $B_{\text {com }} K \rightarrow B_{\text {com }} G$ induces a morphism of the corresponding fibrations:

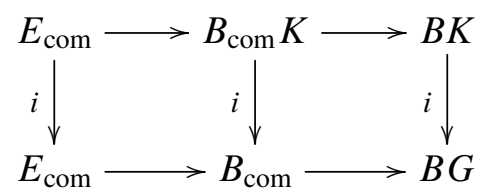

This diagram induces a commutative diagram between the corresponding long exact sequences in homotopy groups. Since the inclusion maps $i: B_{\mathrm{com}} K \rightarrow B_{\mathrm{com}} G$ and $i: B K \rightarrow B G$ are homotopy equivalences, by the five lemma it follows that the inclusion map $i: E_{\mathrm{com}} K \rightarrow E_{\mathrm{com}} G$ is also a homotopy equivalence. 
If $G$ is a compact Lie group then it can be given the structure of a real algebraic variety that is reductive by complete reducibility. Let $G_{\mathbb{C}}$ denote its complexification. Then by the previous theorem it follows that $B_{\text {com }} G$ and $B_{\text {com }} G_{\mathbb{C}}$ are homotopy equivalent and similarly for $E_{\mathrm{com}} G$ and $E_{\mathrm{com}} G_{\mathbb{C}}$. This shows that we can work in the category of compact Lie groups without loss of generality whenever we want to study the spaces $B_{\text {com }} G$ and $E_{\text {com }} G$ for a real or complex reductive algebraic group $G$.

Suppose that $G$ is a topological group; $\operatorname{Hom}\left(\mathbb{Z}^{n}, G\right)$ may fail to be path-connected even if we assume that $G$ is path-connected or simply connected. For every $n \geq 0$ define $\operatorname{Hom}\left(\mathbb{Z}^{n}, G\right)_{1}$ to be the path-connected component of $\operatorname{Hom}\left(\mathbb{Z}^{n}, G\right)$ containing the trivial representation 1: $\mathbb{Z}^{n} \rightarrow G$. It is easy to see that the collection $\left\{\operatorname{Hom}\left(\mathbb{Z}^{n}, G\right)_{1}\right\}_{n \geq 0}$ forms a simplicial subspace of $\left[B_{\mathrm{com}} G\right]_{*}$. We denote by $B_{\mathrm{com}} G_{\mathbf{1}}$ its geometric realization. When $G$ is a compact Lie group the path-connected component $\operatorname{Hom}\left(\mathbb{Z}^{n}, G\right)_{1}$ has the following important feature as already pointed out in [8, Lemma 4.2]. An $n$-tuple $\left(g_{1}, \ldots, g_{n}\right)$ of elements in $G$ belongs to $\operatorname{Hom}\left(\mathbb{Z}^{n}, G\right)_{1}$ if and only if there is a maximal torus $T \subset G$ that contains $g_{1}, \ldots, g_{n}$. On the other hand, if $G$ is a complex reductive algebraic variety then a commuting tuple $\left(g_{1}, \ldots, g_{n}\right)$ belongs to $\operatorname{Hom}\left(\mathbb{Z}^{n}, G\right)_{1}$ if and only if there is a torus $T \subset G$ containing the semisimple part of the Jordan decomposition of $g_{i}$ for all $1 \leq i \leq n$. The spaces $B_{\text {com }} G$ and $B_{\text {com }} G_{\mathbf{1}}$ agree if $\operatorname{Hom}\left(\mathbb{Z}^{n}, G\right)$ is path-connected for all $n \geq 0$. When $G$ is a compact Lie group this is the case if and only if a subgroup $A \subset G$ is a maximal abelian subgroup in $G$ if and only if $A$ is a maximal torus in $G$ by [4, Proposition 2.5]. This is true for Lie groups that arise as finite cartesian products of the groups $\mathrm{SU}(r), U(q)$ and $\operatorname{Sp}(k)$ by [9, Theorem 5.2] and thus $\operatorname{Hom}\left(\mathbb{Z}^{n}, G\right)$ is path-connected for every $n \geq 0$. The same is true for their corresponding complexifications $\mathrm{SL}_{r}(\mathbb{C}), \mathrm{GL}_{q}(\mathbb{C})$ and $\mathrm{Sp}_{k}(\mathbb{C})$. Thus $B_{\mathrm{com}} G=B_{\mathrm{com}} G_{\mathbf{1}}$ for such groups. Note that the argument provided in Theorem 3.1 works exactly in the same way if we replace $B_{\text {com }} G$ by $B_{\text {com }} G_{1}$. Thus if $G$ is a real or complex reductive algebraic group and $K \subset G$ is a maximal compact subgroup then $B_{\text {com }} K_{\mathbf{1}}$ is homotopy equivalent to $B_{\text {com }} G_{\mathbf{1}}$. On the other hand, define $E_{\text {com }} G_{1}:=p^{-1}\left(B_{\text {com }} G_{1}\right)$. Note that $E_{\text {com }} G_{1}$ is the geometric realization of the simplicial subspace of $\left[E_{\mathrm{com}} G\right]_{*}$ defined by $\left[E_{\mathrm{com}} G_{1}\right]_{n}=\operatorname{Hom}\left(\mathbb{Z}^{n}, G\right)_{\mathbf{1}} \times G$. We have a commutative diagram:

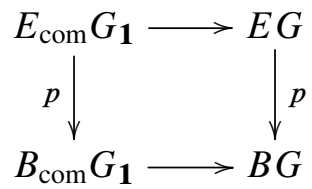

Here the lower horizontal map is the inclusion map $i: B_{\text {com }} G_{\mathbf{1}} \rightarrow B G$. After replacing $i$ with a fibration we obtain a fibration sequence $E_{\mathrm{com}} G_{1} \rightarrow B_{\mathrm{com}} G_{1} \rightarrow B G$ and in 
the same way as was done in Theorem 3.1, we can prove that if $G$ is a real or complex reductive algebraic group and $K \subset G$ is a maximal compact subgroup then $E_{\text {com }} K_{\mathbf{1}}$ is homotopy equivalent to $E_{\mathrm{com}} G_{\mathbf{1}}$.

If $G$ is a connected topological group, the long exact sequence in homotopy groups associated to the fibration sequence $G \rightarrow E G \rightarrow B G$ can be used to show that $B G$ is simply connected. Moreover, if $G$ is a simply connected Lie group, then $G$ is 2-connected since $\pi_{2}(G)=0$ for any Lie group $G$ (see [10, page 225] for the compact case). Thus $B G$ is 3-connected for any such group. As a consequence of [13, Theorem 1.1] similar statements are also true for $B_{\text {com }} G$ and $B_{\text {com }} G_{1}$ as is proved next.

Proposition 3.2 Suppose that $G$ is a real or complex reductive algebraic group that is connected as a topological space. Then $B_{\mathrm{com}} G$ and $B_{\mathrm{com}} G_{\mathbf{1}}$ are simply connected. Moreover, if $G$ is simply connected then $B_{\text {com }} G_{1}$ is 3 -connected.

Proof By Theorem 3.1 we only need to prove the theorem for a compact connected Lie group. Also, by Proposition A.1 in the appendix for any Lie group $G$ the simplicial space $\left[B_{\mathrm{com}} G\right]_{*}$ is a proper simplicial space; in fact it is a strictly proper simplicial space (see Remark A.2). The same is true for $\left[B_{\mathrm{com}} G_{\mathbf{1}}\right]_{*}$. If $G$ is a connected Lie group then $\left[B_{\mathrm{com}} G\right]_{0}=\left[B_{\mathrm{com}} G_{1}\right]_{0}=*$ is in particular 1 -connected and $\left[B_{\mathrm{com}} G\right]_{1}=$ $\left[B_{\text {com }} G_{1}\right]_{1}=G$ is 0 -connected. By [18, Theorem 11.12] it follows that $B_{\text {com }} G$ and $B_{\text {com }} G_{1}$ are simply connected. Suppose now $G$ is simply connected. Then $\left[B_{\mathrm{com}} G_{\mathbf{1}}\right]_{0}=*$ is in particular 3 -connected, $\left[B_{\mathrm{com}} G_{\mathbf{1}}\right]_{1}=G$ and thus this space is 2-connected since $G$ is simply connected and thus 2-connected as pointed out before. Also, $\left[B_{\text {com }} G_{1}\right]_{2}=\operatorname{Hom}\left(\mathbb{Z}^{2}, G\right)_{1}$ is 1 -connected as it is path-connected and simply connected by $\left[13\right.$, Theorem 1.1]. Finally, $\left[B_{\mathrm{com}} G\right]_{3}=\operatorname{Hom}\left(\mathbb{Z}^{3}, G\right)_{1}$ is path-connected by definition. Using [18, Theorem 11.12] it follows that $B_{\text {com }} G_{\mathbf{1}}$ is 3 -connected in this case.

Suppose now that $G$ is a Lie group that arises as a finite product of the classical groups $\mathrm{SU}(r), U(q)$ and $\operatorname{Sp}(k)$. For such groups $E_{\text {com }} G_{\mathbf{1}}=E_{\mathrm{com}} G$ since $\operatorname{Hom}\left(\mathbb{Z}^{n}, G\right)$ is path-connected for all $n \geq 0$ for such groups. Moreover, we have the following.

Proposition 3.3 Assume that $G$ is a Lie group isomorphic to a finite product of the classical groups $\mathrm{SU}(r), U(q)$ and $\mathrm{Sp}(k)$ for $r, q, k \geq 1$. Then $E_{\mathrm{com}} G$ is 3-connected.

Proof Observe that if $G$ and $H$ are topological groups we have a natural homeo$\operatorname{morphism} \operatorname{Hom}\left(\mathbb{Z}^{n}, G \times H\right) \cong \operatorname{Hom}\left(\mathbb{Z}^{n}, G\right) \times \operatorname{Hom}\left(\mathbb{Z}^{n}, H\right)$ for every $n \geq 0$. This implies that there is a homeomorphism $E_{\text {com }}(G \times H) \cong E_{\text {com }} G \times E_{\text {com }} H$. Because 
of this we only need to prove the proposition when $G$ is one of the groups $\operatorname{SU}(r)$, $U(q)$ and $\mathrm{Sp}(k)$. For the groups $\mathrm{SU}(r)$ and $\mathrm{Sp}(k)$ the proposition follows from the previous proposition and Corollary 2.3. Thus we only need to prove the proposition for the case $G=U(q)$. By Proposition 3.2 and Corollary 2.3 it follows that $E_{\text {com }} G$ is simply connected. Hence to show that $E_{\mathrm{com}} G$ is 3-connected it suffices to prove that $\widetilde{H}_{n}\left(E_{\mathrm{com}} G\right)=0$ for $0 \leq n \leq 3$. To see this, recall that the natural filtration of $E_{\mathrm{com}} G$ as the geometric realization of the simplicial space $\left[E_{\mathrm{com}} G\right]_{*}$ induces a spectral sequence

$$
E_{p, q}^{2}=H_{p} H_{q}\left(\left[E_{\mathrm{com}} G\right]_{*}\right) \Rightarrow H_{p+q}\left(E_{\mathrm{com}} G ; \mathbb{Z}\right) .
$$

The term $E_{p, q}^{2}$ in this spectral is obtained by taking the $p^{\text {th }}$ homology group of the simplicial group $H_{q}\left(\left[E_{\mathrm{com}} G\right]_{*}\right)$. Trivially we have $E_{0,0}^{2}=\mathbb{Z}$. We show next that $E_{p, q}^{2}=0$ for all $p, q \geq 0$ with $0<p+q \leq 3$. To prove this, we claim that the map of simplicial spaces $i_{*}:\left[E_{\mathrm{com}} G\right]_{*} \rightarrow[E G]_{*}$ induced by the inclusion map induces an isomorphism $i_{*}: H_{p} H_{q}\left(\left[E_{\mathrm{com}} G\right]_{*}\right) \rightarrow H_{p} H_{q}\left([E G]_{*}\right)$ for $0 \leq p+q \leq 3$. Since $H_{p} H_{q}\left([E G]_{*}\right)=0$ for all $p+q>0$ then the proposition follows. The claim is trivial for $q=0$ because $\left[E_{\mathrm{com}} G\right]_{k}$ is connected for all $k \geq 0$. When $q=1$ the simplicial groups $H_{1}\left(\left[E_{\mathrm{com}} G\right]_{*}\right)$ and $H_{1}\left([E G]_{*}\right)$ are isomorphic by [13, Theorem 1.1]. Suppose now that $q=2$. Let $C_{n}=H_{2}\left(\operatorname{Hom}\left(\mathbb{Z}^{n}, G\right) \times G ; \mathbb{Z}\right)$ so that $\left\{C_{n}\right\}_{n \geq 0}$ is the chain complex whose $p^{\text {th }}$ homology is $H_{p} H_{2}\left(\left[E_{\mathrm{com}} G\right]_{*}\right)$. Trivially we have that $C_{0}=0$ since $H_{2}(G ; \mathbb{Z})=0$. Also, $C_{1} \cong \mathbb{Z}$ and the differential $\partial: C_{2} \rightarrow C_{1} \cong \mathbb{Z}$ is surjective since the inclusion $G \vee G \hookrightarrow \operatorname{Hom}\left(\mathbb{Z}^{2}, G\right)$ induces a split injection at the level of homology by [1, Theorem 1.6]. This shows that $H_{p} H_{2}\left([E G]_{*}\right)=0$ for $p=0,1$. Finally, $\mathrm{H}_{0} \mathrm{H}_{3}\left(\left[E_{\mathrm{com}} G\right]_{*}\right)$ vanishes trivially.

\section{Commutative K-theory}

Suppose that $X$ is a finite CW-complex and let $p: E \rightarrow X$ be an $n$-plane complex vector bundle. As in the case of a principal bundles, we say that $E$ is transitionally commutative if we can find an open cover $\left\{U_{i}\right\}_{i \in I}$ of $X$ such that $E$ is trivial over each $U_{i}$ and the corresponding transition functions commute with each other whenever they are simultaneously defined. This is equivalent to saying that, with a Hermitian metric in sight, the corresponding frame bundle is a transitionally commutative principal $U(n)-$ bundle. Similarly, two such complex vector bundles $q_{0}: E_{0} \rightarrow X$ and $q_{1}: E_{1} \rightarrow X$ are said to be transitionally commutative isomorphic if their frame bundles are transitionally commutative isomorphic. This means that we can find a transitionally commutative vector bundle $p: E \rightarrow X \times[0,1]$ such that $q_{0}=p_{\mid p^{-1}(X \times\{0\})}$ and $q_{1}=p_{\mid p^{-1}(X \times\{1\})}$. Let $\operatorname{Vect}_{\text {com }}(X)$ be the set of transitionally commutative isomorphism classes of transitionally commutative vector bundles over $X$. The Whitney sum of two transitionally 
commutative vector bundles is also transitionally commutative and thus $\operatorname{Vect}_{\text {com }}(X)$ has the structure of a monoid. We define the commutative $\mathrm{K}$-theory of $X$ to be $K_{\text {com }}(X):=\operatorname{Gr}\left(\operatorname{Vect}_{\text {com }}(X)\right)$, where Gr denotes the Grothendieck construction. It is easy to see that if $E$ and $F$ are two transitionally commutative vector bundles over $X$, then $E \oplus F$ is transitionally commutative isomorphic to $F \oplus E$. This shows that, as in the case of classical K-theory, this construction defines a functor from the category of topological spaces to the category of abelian groups.

By Theorem 2.2 any transitionally commutative $n$-plane complex vector bundle is classified by a map $f: X \rightarrow B_{\text {com }} U(n)$. Moreover, two such vector bundles classified by maps $f, g: X \rightarrow B_{\text {com }} U(n)$ are transitionally commutative isomorphic if and only if $f$ is homotopic to $g$. Let $U=\operatorname{colim}_{n \rightarrow \infty} U(n)$, where the colimit is taken over the natural inclusions $i_{n}: U(n) \rightarrow U(n+1)$. We conclude, in an analogous way to the case of $\mathrm{K}$-theory, that there is a natural isomorphism of groups $K_{\text {com }}(X) \cong\left[X, \mathbb{Z} \times B_{\text {com }} U\right]$.

Theorem 4.1 The space $\mathbb{Z} \times B_{\text {com }} U$ is a loop space and for any finite $C W$-complex $X$ there is a natural isomorphism of groups $K_{\text {com }}(X) \cong\left[X, \mathbb{Z} \times B_{\text {com }} U\right]$.

Proof As pointed out above we have a natural isomorphism $K_{\text {com }}(X) \cong\left[X, \mathbb{Z} \times B_{\text {com }} U\right]$ for any finite CW-complex $X$. Consider $M:=\bigsqcup_{n \geq 0} B_{\text {com }} U(n)$; this space has the structure of a topological monoid defined as follows. For each $n, m \geq 0$ consider the homomorphism of topological groups

$$
\begin{aligned}
\iota_{n, m}: U(n) \times U(m) & \rightarrow U(n+m), \\
(A, B) & \mapsto\left[\begin{array}{cc}
A & 0 \\
0 & B
\end{array}\right] .
\end{aligned}
$$

This homomorphism induces a continuous map

$$
\Gamma_{n, m}: B_{\mathrm{com}} U(n) \times B_{\text {com }} U(m)=B_{\mathrm{com}}(U(n) \times U(m)) \rightarrow B_{\mathrm{com}} U(n+m) .
$$

The different maps $\left\{\Gamma_{n, m}\right\}_{n, m \geq 0}$ can be assembled to obtain a map $\Gamma: M \times M \rightarrow M$ giving $M$ the structure of a strictly associative topological monoid. Moreover, this monoid is commutative up to homotopy. Indeed, for each $n, m \geq 0$ fix a continuous path $\beta_{n, m}:[0,1] \rightarrow U(n+m)$ from the identity matrix $I_{n+m}$ to the matrix $\left[\begin{array}{cc}0 & I_{m} \\ I_{n} & 0\end{array}\right]$. Such a path exists because $U(n+m)$ is path-connected. These paths induce a continuous family of homomorphisms

$$
\begin{aligned}
h_{n, m}(t): U(n) \times U(m) & \rightarrow U(n+m), \\
(A, B) & \mapsto \beta_{n, m}(t) \iota_{n, m}(A, B) \beta_{n, m}(t)^{-1}
\end{aligned}
$$

defined for $0 \leq t \leq 1$. After applying the functor $B_{\text {com }}$, these maps induce a homotopy $h: M \times M \times I \rightarrow M$ such that $h(A, B, 0)=\Gamma(A, B)$ and $h(A, B, 1)=\Gamma(B, A)$. The 
above proves that $M$ is a strict topological monoid that is commutative up to homotopy. On the other hand, observe that $\pi_{0}(M)=\mathbb{N}$. Fix an element $m \in B_{\text {com }} U(1)$ and consider the mapping telescope

$$
M_{\infty}=\operatorname{Tel}(M \stackrel{* m}{\longrightarrow} M \stackrel{* m}{\longrightarrow} M \stackrel{* m}{\longrightarrow} \cdots) \cong \mathbb{Z} \times B_{\mathrm{com}} U .
$$

By the group completion theorem (see for example [21, Proposition 1]), it follows that the natural map $M \rightarrow \Omega B M$ induces a map $\eta: M_{\infty} \cong \mathbb{Z} \times B_{\text {com }} U \rightarrow \Omega B M$ that is an isomorphism in homology. Let $(\Omega B M)_{0}$ be the path-connected component of $\Omega B M$ containing the trivial loop. Then the restriction of $\eta, \eta_{0}: B_{\text {com }} U \rightarrow(\Omega B M)_{0}$, induces an isomorphism in homology with integer coefficients. By Proposition 3.2 the space $B_{\text {com }} U(n)$ is simply connected for every $n \geq 0$. Since $B_{\text {com }} U=\operatorname{colim}_{n \geq 0} B_{\text {com }} U(n)$ the same is true for $B_{\text {com }} U$. Similarly $(\Omega B M)_{0}$ is simply connected. Therefore $\eta_{0}: B_{\text {com }} U \rightarrow(\Omega B M)_{0}$ is a homology isomorphism between simply connected spaces. By the Hurewicz theorem we conclude that $\eta_{0}$ is a homotopy equivalence. On the other hand, since $\Omega B M$ is a loop space, all of its connected component are homotopy equivalent. We conclude that there is a homotopy equivalence $\mathbb{Z} \times B_{\text {com }} U \simeq \Omega B M$ and thus $\mathbb{Z} \times B_{\text {com }} U$ is a loop space.

Proposition 4.2 If $X$ is a connected finite $C W$-complex, there is a natural isomorphism of groups $K_{\text {com }}(\Sigma X) \cong K^{0}(\Sigma X) \times\left[\Sigma X, E_{\text {com }} U\right]$.

Proof By [3, Theorem 6.3], for any $n \geq 0$ there is a natural homotopy equiv-

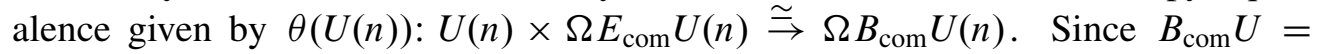
$\operatorname{colim}_{n \rightarrow \infty} B_{\text {com }} U(n)$ and $E_{\text {com }} U=\operatorname{colim}_{n \rightarrow \infty} E_{\text {com }} U(n)$, by passing to the colimit as $n \rightarrow \infty$ we obtain a homotopy equivalence $\Omega B_{\text {com }} U \simeq U \times \Omega E_{\text {com }} U$. Using this homotopy equivalence and adjunction between the functors $\Sigma$ and $\Omega$, we obtain natural isomorphisms

$$
\begin{aligned}
K_{\mathrm{com}}(\Sigma X) & \cong\left[\Sigma X, \mathbb{Z} \times B_{\mathrm{com}} U\right] \cong \mathbb{Z} \times\left[\Sigma X, B_{\mathrm{com}} U\right] \\
& \cong \mathbb{Z} \times\left[X, U \times \Omega E_{\mathrm{com}} U\right] \cong \mathbb{Z} \times[X, U] \times\left[X, \Omega E_{\mathrm{com}} U\right] \\
& \cong[\Sigma X, \mathbb{Z} \times B U] \times\left[X, \Omega E_{\mathrm{com}} U\right] \cong K^{0}(\Sigma X) \times\left[\Sigma X, E_{\mathrm{com}} U\right] .
\end{aligned}
$$

Example 4.3 Using this proposition we see that $K_{\text {com }}\left(\mathbb{S}^{m}\right) \cong K^{0}\left(\mathbb{S}^{m}\right)$ for $0 \leq m \leq 3$. For $m=0$ this is trivial; for $1 \leq m \leq 3$, by the above computation there is an isomorphism $K_{\text {com }}\left(\mathbb{S}^{m}\right) \cong K^{0}\left(\mathbb{S}^{m}\right) \times\left[\mathbb{S}^{m}, E_{\text {com }} U\right]$. The space $E_{\text {com }} U(n)$ is 3-connected by Proposition 3.3 for all $n \geq 0$. By passing to the colimit as $n \rightarrow \infty$ it follows that the same is true for $E_{\text {com }} U$. Therefore for $1 \leq m \leq 3$ we have that $\pi_{m}\left(E_{\text {com }} U\right) \cong\left[\mathbb{S}^{m}, E_{\text {com }} U\right]$ is trivial and we conclude that $K_{\text {com }}\left(\mathbb{S}^{m}\right) \cong K^{0}\left(\mathbb{S}^{m}\right)$ for $0 \leq m \leq 3$. However, $K_{\text {com }}\left(\mathbb{S}^{4}\right) \not K^{0}\left(\mathbb{S}^{4}\right)$. To see this note that the cohomological computations derived in 
Section 8 imply that $H^{4}\left(E_{\text {com }} U ; \mathbb{Q}\right) \neq 0$. This together with the universal coefficient theorem and the Hurewicz theorem imply that $\pi_{4}\left(E_{\mathrm{com}} U\right) \cong\left[\mathbb{S}^{4}, E_{\mathrm{com}} U\right] \neq 0$ and thus $K_{\text {com }}\left(\mathbb{S}^{4}\right) \not K^{0}\left(\mathbb{S}^{4}\right)$. This in particular shows that the functor $K_{\text {com }}$ does not satisfy Bott periodicity for its values on spheres. We should also mention that the nontrivial element in $\pi_{4}\left(B_{\text {com }} \mathrm{SU}(2)\right)$, which we described in Example 2.5, is mapped nontrivially to $\pi_{4}\left(B_{\mathrm{com}} U(2)\right)$ and this defines a nontrivial commutative vector bundle over $\mathbb{S}^{4}$, which is trivial as an ordinary bundle.

Remark 4.4 In [5] it is proved that $\mathbb{Z} \times B_{\text {com }} U$ is in fact an infinite loop space. In particular it follows that the definition of commutative $\mathrm{K}$-theory can be extended to obtain a generalized cohomology theory. Moreover, it is shown there that the fibration sequence $E_{\text {com }} U \rightarrow B_{\text {com }} U \rightarrow B U$ splits and that $\mathbb{Z} \times B_{\text {com }} U \simeq(\mathbb{Z} \times B U) \times E_{\text {com }} U$ as infinite loop spaces. This implies in particular that commutative $\mathrm{K}$-theory contains topological K-theory as a summand. Note however that, as proved in Example 4.3, commutative $\mathrm{K}$-theory is not 2-periodic, unlike classical $\mathrm{K}$-theory. The homotopy type of $\mathbb{Z} \times B_{\text {com }} U$ remains to be determined.

\section{The topological poset associated to maximal tori in a Lie group}

Our next goal is to provide a description of the spaces $B_{\text {com }} G_{1}$ as suitable homotopy colimits for any Lie groups $G$ that are compact and connected. To achieve this decomposition we first need to study the poset generated by all maximal tori in a compact Lie group.

We begin by recalling the definition of a topological poset.

Definition 5.1 A topological poset is a partially ordered set $(\mathcal{R}, \preceq)$ together with a topology on the set of objects $\mathcal{R}$ in such a way that the order space $\mathcal{O}_{\mathcal{R}}:=\{(x, y) \in$ $\mathcal{R} \times \mathcal{R} \mid x \preceq y$ \} is a closed subset of $\mathcal{R} \times \mathcal{R}$.

A topological poset can be seen as a topological category where the space of objects is $\mathcal{R}$ and the space of morphisms is the order space $\mathcal{O}_{\mathcal{R}}$. In this article, by a topological category we mean a small category $\mathcal{D}$ for which the sets $\operatorname{Ob}(\mathcal{D})$ and $\operatorname{Mor}(\mathcal{D})$ come equipped with topologies in such a way that the structural maps source, target, composition and identity are continuous maps.

Example 5.2 Let $n \geq 0$ be a fixed integer. Given $0 \leq k \leq n$, denote by $G_{k}\left(\mathbb{C}^{n}\right)$ the Grassmannian manifold consisting of all those $k$-dimensional $\mathbb{C}$-vector spaces in $\mathbb{C}^{n}$. 
Let $\mathcal{G} r(n)$ be the poset of all $\mathbb{C}$-vector subspaces in $\mathbb{C}^{n}$. This set naturally has the structure of a poset by inclusion. Note that $\mathcal{G} r(n)=\bigsqcup_{0 \leq k \leq n} G_{k}\left(\mathbb{C}^{n}\right)$. We can use this identification to give $\mathcal{G} r(n)$ a topology making it into a topological poset.

The maximal tori in a compact Lie group $G$ define a topological poset in the following natural way.

Definition 5.3 Suppose that $G$ is a Lie group. Define $\mathcal{T}(G)$ to be the poset whose objects are closed subspaces $S \subset G$ arising as the intersection of a collection of maximal tori in $G$, with the order in $\mathcal{T}(G)$ given by inclusion.

The set $\mathcal{T}(G)$ can be given a topology making it into a topological poset as follows. Let $\mathcal{C}(G)$ denote the set of all closed subspaces in $G$. Suppose that $\mathcal{U}:=\left\{U_{1}, \ldots, U_{n}\right\}$ is a finite collection of open sets in $G$. Define $\mathcal{C}(G, \mathcal{U})$ to be the set of elements $A \in \mathcal{C}(G)$ such that $A \subset \bigcup_{i=1}^{n} U_{i}$ and $A \cap U_{i} \neq \varnothing$ for all $1 \leq i \leq n$. The sets of the form $\mathcal{C}(G, \mathcal{U})$ form a basis for a topology in $\mathcal{C}(G)$ called the finite topology (see [22] for details). Note that $\mathcal{T}(G) \subset \mathcal{C}(G)$ and in this way we can give $\mathcal{T}(G)$ the subspace topology making it into a topological poset. Our next goal is to describe the structure of $\mathcal{T}(G)$ as a topological space for any compact connected Lie group $G$.

Let $\mathfrak{g}$ denote the Lie algebra of $G$ and fix $T \subset G$ a maximal torus in $G$ with Lie algebra $\mathfrak{t}$. Let $\Phi$ be the root system associated to $(\mathfrak{g}, \mathfrak{t})$ and fix a subset $\Phi^{+}$of positive roots of $\Phi$. For each $\alpha \in \Phi^{+}$and any integer $n$, define

$$
\mathfrak{t}_{\alpha}:=\{X \in \mathfrak{t} \mid \alpha(X) \in 2 \pi i \mathbb{Z}\}, \quad \mathfrak{t}_{\alpha, n}:=\{X \in \mathfrak{t} \mid \alpha(X)=2 \pi i n\} .
$$

Each $\mathfrak{t}_{\alpha, n}$ is a hyperplane of codimension one and the set $D(G):=\bigcup_{\alpha \in \Phi^{+}} \mathfrak{t}_{\alpha}$ is called the Stiefel diagram of $G$. Recall that an element $g \in G$ is called singular if it belongs to more than one maximal torus in $G$. Equivalently, $g \in G$ is singular if and only if $\operatorname{dim}\left(Z_{G}(g)\right)>\operatorname{dim}(T)$. Let $G_{s} \subset G$ be the set of singular elements in $G$ and let $T_{s}=T \cap G_{s}$ be the set of singular elements in $T$. Consider the restriction of the exponential map exp: $\mathfrak{t} \rightarrow T$. We have $\exp ^{-1}\left(T_{S}\right)=D(G)$; that is, if $X \in \mathfrak{t}$ then $\exp (X)$ is singular if and only if $X \in D(G)$. Given a set of positive roots $I=\left\{\alpha_{1}, \ldots, \alpha_{k}\right\}$ define

$$
t_{I}=\bigcap_{i=1}^{k} t_{\alpha_{i}} \quad \text { and } \quad T_{I}:=\exp \left(t_{I}\right) \subset T .
$$

In the previous definition we allow the case $k=0$. In this case we take the convention that $t_{\varnothing}=\mathfrak{t}$ and thus $T_{\varnothing}=T$. Let $\Delta=\left\{\alpha_{1}, \ldots, \alpha_{r}\right\}$ be a set of simple roots for the root system $\Phi$. Recall that the Weyl group $W$ is a reflection subgroup generated by 
the reflections $s_{\alpha}$ corresponding to elements $\alpha \in \Delta$. Given $I \subset \Delta$, the subgroup of $W_{I}$ of $W$ generated by the reflections $s_{\alpha}$ corresponding to elements $\alpha \in I$ is called a parabolic subgroup of $W$. Note that each parabolic subgroup is itself a reflection subgroup. We are interested in the different parabolic subgroups of $W$ up to conjugacy. If $I, J \subset \Delta$, then $W_{I}$ is conjugated to $W_{J}$ if and only if $I=w J$ for some $w \in W$. In that case we say that $I$ and $J$ are in the same Coxeter class and write $I \sim_{W} J$. The relation $\sim_{W}$ defines an equivalence relation on the set of subsets of $\Delta$. We denote by $\mathcal{E}_{W}$ the set of equivalence classes of subsets of $\Delta$ under this equivalence relation and by $[I]$ the equivalence class in $\mathcal{E}_{W}$ that contains $I \subset \Delta$.

Theorem 5.4 Suppose that $G$ is a compact connected Lie group. Fix $\Delta=\left\{\alpha_{1}, \ldots, \alpha_{r}\right\}$ a set of simple roots. Then any element $S \in \mathcal{T}(G)$ is conjugated to $T_{I}$ for some $I \subset \Delta$. Moreover, there is a $G$-equivariant homeomorphism $\mathcal{T}(G) \cong \bigsqcup_{[I] \in \mathcal{E}_{W}} G / N_{G}\left(T_{I}\right)$.

Proof Fix a maximal torus $T \subset G$ and suppose that $S \in \mathcal{T}(G)$. Since any two maximal tori in $G$ are conjugated, after replacing $S$ with a suitable conjugate we may assume that $S \subset T$. If $S=T$, then $S=T_{\varnothing}$ and there is nothing to prove. Assume then that $S \subsetneq T$. We will show that under this assumption $S$ is conjugated to $T_{I}$ for some set of simple roots $I \subset \Delta$. Let $S_{0}$ be the identity component of $S$. Then $S_{0}$ is a compact, connected and abelian subgroup of $G$. Thus $S_{0}$ is a torus and in particular we can find an element $x_{0} \in S_{0}$ such that $S_{0}=\overline{\left\langle x_{0}\right\rangle}$. Let $\beta_{1}, \ldots, \beta_{l}$ be the set of positive roots $\alpha$ with $x_{0} \in T_{\alpha}$. It follows that $S_{0} \subset T_{\beta_{1}} \cap \cdots \cap T_{\beta_{l}}=T_{J}$, where $J=\left\{\beta_{1}, \ldots, \beta_{l}\right\}$. In fact $S_{0} \subset T_{J, 0}$, where $T_{J, 0}$ denotes the identity component of $T_{J}$. As a first step we show that $S_{0}=T_{J, 0}$. To see this recall that the adjoint representation provides a decomposition of the complexification of $\mathfrak{g}$ into a direct sum of weight spaces $\mathfrak{g}_{\mathbb{C}}=\mathfrak{t}_{\mathbb{C}} \oplus\left(\bigoplus_{\alpha \in \Phi} L_{\alpha}\right)$. This in turn provides a decomposition of $\mathfrak{g}$ in the form $\mathfrak{g}=\mathfrak{t} \oplus\left(\bigoplus_{\alpha \in \Phi^{+}} M_{\alpha}\right)$, where $M_{\alpha}=\left(L_{\alpha} \oplus L_{-\alpha}\right) \cap \mathfrak{g}$. Note that $Z_{G}\left(x_{0}\right)=Z_{G}\left(S_{0}\right)$ and in particular this group is connected since the centralizer of any torus in $G$ is connected. By [10, Proposition V 2.3] the Lie algebra of $Z_{G}\left(x_{0}\right)$ is $\mathfrak{z}_{\mathfrak{g}}\left(x_{0}\right)=\mathfrak{t} \oplus\left(\bigoplus_{i=1}^{l} M_{\beta_{i}}\right)$. On the other hand, the Lie algebra of $T_{J, 0}$ is $\mathfrak{t}_{J, 0}:=\mathfrak{t}_{\beta_{1}, 0} \cap \cdots \cap \mathfrak{t}_{\beta_{l}, 0}$. It follows that the Lie algebra of $Z_{G}\left(T_{J, 0}\right)$ is $\mathfrak{z}_{\mathfrak{g}}\left(\mathfrak{t}_{J, 0}\right)=\mathfrak{t} \oplus\left(\bigoplus_{i=1}^{l} M_{\beta_{i}}\right)$. This proves that $Z_{G}\left(x_{0}\right)$ and $Z_{G}\left(T_{J, 0}\right)$ are connected subgroups of $G$ that have the same Lie algebra, which in turn implies that $Z_{G}\left(x_{0}\right)=Z_{G}\left(T_{J, 0}\right)$. Suppose now that $T^{\prime}$ is a maximal torus that contains $x_{0}$. Then $T^{\prime} \subset Z_{G}\left(x_{0}\right)=Z_{G}\left(T_{J, 0}\right)$, which implies that $T_{J, 0} \subset T^{\prime}$ since the centralizer of a connected abelian subgroup of $G$ is the union of all maximal tori containing it. This shows that $T_{J, 0}$ is contained in the intersection of all maximal tori that contain $x_{0}$. Since $S$ is the intersection of a family of maximal tori, it follows that $T_{J, 0} \subset S$, and by connectedness we have $T_{J, 0} \subset S_{0}$. We conclude that $S_{0}=T_{J, 0}$. To show that $T_{J}=S$ recall that the center of $G$ is the intersection of all maximal tori in $G$. 
Therefore $S$ contains the center of $G$. This is also true for $T_{J}$. Moreover, it is easy to see that the center of $G$ intersects all the connected components of $S$ and $T_{J}$. From here it follows that $S=T_{J}$ and that the Lie algebra of $S$ is $\mathfrak{t}_{J, 0}$. We will show now that after replacing $S$ with some further conjugate, we can choose the $\beta_{j}$ to be simple roots. Choose a minimal set of positive roots $\gamma_{1}, \ldots, \gamma_{k}$ with $\mathfrak{t}_{J, 0}=\mathfrak{t}_{\gamma_{1}, 0} \cap \cdots \cap \mathfrak{t}_{\gamma_{k}, 0}$. Then we have proper inclusions $\mathfrak{t}_{\gamma_{1}, 0} \cap \cdots \cap \mathfrak{t}_{\gamma_{k}, 0} \subset \mathfrak{t}_{\gamma_{1}, 0} \cap \cdots \cap \mathfrak{t}_{\gamma_{k-1}, 0} \subset \cdots \subset \mathfrak{t}_{\gamma_{1,0}}$. As $\mathfrak{t} \backslash \bigcup_{\alpha \in \Phi^{+}} \mathfrak{t}_{\alpha, 0}$ is a union of Weyl chambers, then we can find some (closed) Weyl chamber $\mathfrak{C}$ in such a way that each $\mathfrak{t}_{\gamma_{i}, 0}$ is a face of $\mathfrak{C}$ for every $1 \leq i \leq k$. Associated to the Weyl chamber $\mathfrak{C}$ there is a set of simple roots of $\Phi$. Since each $\mathfrak{t}_{\gamma_{i}, 0}$ is a face of the chamber $\mathfrak{C}$ then, after replacing the sign of the $\gamma_{i}$ if necessary, the roots $\gamma_{1}, \ldots, \gamma_{k}$ are roots in some base of $\Phi$. Since the Weyl group acts transitively on the set of all bases on $\Phi$, it follows that we can find some $w \in W$ such that $w \gamma_{1}, \ldots, w \gamma_{k}$ are in $\Delta$. This shows that $S$ is conjugated to $T_{I}$, where $I=\left\{\alpha_{i_{1}}, \ldots, \alpha_{i_{k}}\right\}$ is some set of simple roots.

On the other hand, it is easy to see that for any $I \subset \Delta$, the closed subspace $T_{I}$ is the intersection of all the maximal tori containing it and thus $T_{I} \in \mathcal{T}(G)$. Also, if $I$ and $J$ are subsets of $\Delta$, then $T_{I}$ is conjugated to $T_{J}$ if and only if $J=w I$ for some $w \in W$; that is, $T_{I}$ is conjugated to $T_{J}$ if and only if $I$ and $J$ are in the same Coxeter class. To finish note that the space of subgroups in $G$ that are conjugated to $T_{I}$ can be identified with $G / N_{G}\left(T_{I}\right)$ and thus the theorem follows.

Example 5.5 Suppose that $G=U(n)$ for $n \geq 1$. For this group a maximal torus $T$ can be chosen to be the set of all diagonal matrices with diagonal entries $x_{1}, \ldots, x_{n} \in \mathbb{S}^{1}$. The Weyl group $W=\Sigma_{n}$ acts by permutation on the diagonal entries. The Lie algebra $\mathfrak{t}$ can be identified with $\mathfrak{t}=\left\{\left(X_{1}, \ldots, X_{n}\right) \mid X_{i} \in i \mathbb{R}\right.$ for all $\left.1 \leq i \leq n\right\}$. The root system $\Phi$ consists of all functions $\alpha_{i, j}\left(X_{1}, \ldots, X_{n}\right)=X_{i}-X_{j}$ for $1 \leq i, j \leq n$ with $i \neq j$. A choice of positive roots is the set of roots $\alpha_{i, j}$ with $i<j$ and the roots

$$
\Delta:=\left\{\alpha_{1}:=\alpha_{1,2}, \alpha_{2}:=\alpha_{2,3}, \ldots, \alpha_{n-1}:=\alpha_{n-1, n}\right\}
$$

form a set of simple roots. By the previous theorem any $S \in \mathcal{T}(U(n))$ is conjugated to some $T_{I}$, where $I:=\left\{\alpha_{i_{1}}, \ldots, \alpha_{i_{k}}\right\}$ and $1 \leq i_{1}<\cdots<i_{k} \leq n-1$. Unraveling the definition, we see that $T_{I}$ consists of all diagonal matrices with entries $x_{1}, \ldots, x_{n}$ and with $x_{i_{r}}=x_{i_{r}+1}$ for all $1 \leq r \leq k$. In other words, the roots $\alpha_{i_{1}}, \ldots, \alpha_{i_{k}}$ determine the number of repeated diagonal entries in the elements of $T_{I}$. The conjugacy classes of such tori can be parametrized using partitions of the number $n$. Recall that a nondecreasing sequence of integers $\lambda=\left(\lambda_{1}, \ldots, \lambda_{k}\right)$ is a partition of $n$ if $n=\lambda_{1}+\lambda_{2}+\cdots+\lambda_{k}$. We write $\lambda \vdash n$ to mean that $\lambda$ is a partition of $n$. The set of Coxeter classes in $\Delta$ are in bijective correspondence with the set of partitions $\lambda$ of 
the number $n$. This can be seen by corresponding for a partition $\lambda$ of $n$ the Coxeter class represented by the set of simple roots $I(\lambda):=\Delta \backslash\left\{\alpha_{\lambda_{1}}, \ldots, \alpha_{\lambda_{1}+\cdots+\lambda_{k-1}}\right\}$. Note that if $\lambda \vdash n$ then the associated torus $T_{I(\lambda)}$ is the subspace of $T$ consisting of those diagonal matrices with entries of the form

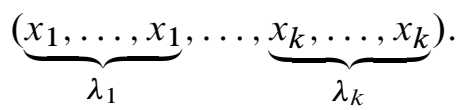

Therefore each $S \in \mathcal{T}(U(n))$ is conjugated to $T_{I(\lambda)}$ for some unique $\lambda \vdash n$. On the other hand, given a partition $\lambda$ of $n$, let $F l(\lambda):=U(n) /\left(U\left(\lambda_{1}\right) \times \cdots \times U\left(\lambda_{k}\right)\right)$. The space $F l(\lambda)$ is the flag manifold consisting of all flags of the form $V_{1} \subset V_{2} \subset \cdots \subset V_{k}=\mathbb{C}^{n}$, where $V_{i}$ is a $\mathbb{C}$-vector subspace of $\mathbb{C}^{n}$ of dimension $\operatorname{dim}_{\mathbb{C}}\left(V_{i}\right)=\lambda_{1}+\cdots+\lambda_{i}$. We can see the partition $\lambda$ as an ordered $k$-tuple $\left(\lambda_{1}, \ldots, \lambda_{k}\right)$. The symmetric group $\Sigma_{k}$ acts on the set of such $k$-tuples by permutation. We denote by $\Sigma_{\lambda}$ the isotropy of $\Sigma_{k}$ at $\lambda$ under this action. With this notation, the group $N_{U(n)}\left(T_{I(\lambda)}\right)$ fits in a short exact sequence

$$
1 \rightarrow U\left(\lambda_{1}\right) \times \cdots \times U\left(\lambda_{k}\right) \rightarrow N_{U(n)}\left(T_{I(\lambda)}\right) \rightarrow \Sigma_{\lambda} \rightarrow 1 .
$$

It follows that if $\lambda \vdash n$, then

$$
U(n) / N_{U(n)}\left(T_{I(\lambda)}\right) \cong\left(U(n) /\left(U\left(\lambda_{1}\right) \times \cdots \times U\left(\lambda_{r}\right)\right)\right) / \Sigma_{\lambda}=F l(\lambda) / \Sigma_{\lambda} .
$$

We conclude that $\mathcal{T}(U(n))=\bigsqcup_{\lambda \vdash n} F l(\lambda) / \Sigma_{\lambda}$, where $\lambda$ runs through all partitions of $n$.

\section{The space $B_{\text {com }} G_{1}$ as a homotopy colimit}

In this section we derive a description of $B_{\text {com }} G_{\mathbf{1}}$ as a suitable homotopy colimit for a real or complex reductive algebraic group $G$. Note that by Theorem 3.1 we can work without loss of generality in the category of compact Lie groups.

To start we show that the space $B_{\text {com }} G_{\mathbf{1}}$ is a colimit over the poset $\mathcal{T}(G)$.

Proposition 6.1 For any compact connected Lie group $G$ we have

$$
B_{\mathrm{com}} G_{\mathbf{1}} \cong \operatorname{colim}_{S \in \mathcal{T}(G)} B S .
$$

Proof Suppose that $T \subset G$ is a maximal torus. Then $T^{n} \subset \operatorname{Hom}\left(\mathbb{Z}^{n}, G\right)_{\mathbf{1}}$ for all $n \geq 0$ and thus $B T \subset B_{\text {com }} G_{\mathbf{1}}$. This proves that $\bigcup_{T \in \mathcal{T}(G)} B T \subset B_{\text {com }} G_{\mathbf{1}}$. Suppose now that $x \in B_{\mathrm{com}} G_{\mathbf{1}}$. Then $x$ can be represented in the form $x=\left[\left(g_{1}, \ldots, g_{n}, t\right)\right]$ for some $\left(g_{1}, \ldots, g_{n}\right) \in \operatorname{Hom}\left(\mathbb{Z}^{n}, G\right)_{1}$ and $t \in \Delta_{n}$. By [8, Lemma 4.2] there is a maximal torus $T \subset G$ such that $g_{i} \in T$ for all $1 \leq i \leq n$. Therefore $x \in B T$. This proves that $B_{\text {com }} G_{\mathbf{1}}=\bigcup_{T \in \mathcal{T}(G)} B T$ and thus $B_{\text {com }} G_{\mathbf{1}}=\bigcup_{T \in \mathcal{T}(G)} B T \cong \operatorname{colim}_{S \in \mathcal{T}(G)} B S$. 
As is well known, homotopy colimits are better suited for homotopical computations than colimits. Therefore we would like to obtain a decomposition $B_{\text {com }} G_{\mathbf{1}}$ as a homotopy colimit over a suitable category. It can be seen that the space $B_{\text {com }} G_{1}$ can be described as the homotopy colimit of the functor $B: \mathcal{T}(G) \rightarrow$ Top. However, this decomposition is not very helpful as the category $\mathcal{T}(G)$ is a topological category (see $[16 ; 17]$ for a discussion of homotopy colimits over topological categories). In particular the usual Bousfield-Kan spectral sequence does not apply to such homotopy colimits. We will get around this issue by obtaining a decomposition of $B_{\text {com }} G_{1}$ as a homotopy colimit over a discrete category. A key element in this decomposition is the rank function defined over the poset $\mathcal{T}(G)$. To be more precise, suppose that $G$ is a compact connected Lie group. Let $Z=Z(G)$ be the center of $G$ and write $n=\operatorname{rank}(G)-\operatorname{rank}(Z) \geq 0$. If $S \in \mathcal{T}(G)$ then $S$ is a closed subgroup of $G$ and in particular it is a compact Lie group. Define

$$
\rho(S):=\operatorname{rank}(S)-\operatorname{rank}(Z) .
$$

This way for every $S \in \mathcal{T}(G)$ we obtain $0 \leq \rho(S) \leq n$.

Proposition 6.2 If $G$ is a compact connected Lie group the function $\rho: \mathcal{T}(G) \rightarrow \mathbb{N}$ is strictly increasing and continuous. Moreover, $\rho$ attains any value $0 \leq m \leq n$.

Proof Suppose that $S_{2} \subsetneq S_{1}$ are elements in $\mathcal{T}(G)$. Fix a maximal torus $T \subset G$ and a set $\Phi^{+}$of positive roots. By Theorem 5.4 we can find simple roots $I:=\left\{\alpha_{i_{1}}, \ldots, \alpha_{i_{k}}\right\}$ and $J:=\left\{\beta_{j_{1}}, \ldots, \beta_{j_{l}}\right\}$ and $g_{1}, g_{2} \in G$ such that $S_{2}=g_{2} T_{J} g_{2}^{-1} \subsetneq S_{1}=g_{1} T_{I} g_{1}^{-1}$. Since the exponential map is surjective this implies $g_{2} \mathfrak{t}_{J} g_{2}^{-1} \subsetneq g_{1} \mathfrak{t}_{I} g_{1}^{-1}$. In fact we must have $g_{2} \mathfrak{t}_{J, 0} g_{2}^{-1} \subsetneq g_{1} \mathfrak{t}_{I, 0} g_{1}^{-1}$. Therefore $\operatorname{rank}\left(S_{2}\right)=\operatorname{dim}_{\mathbb{R}}\left(g_{2} \mathfrak{t}_{J, 0} g_{2}^{-1}\right)<$ $\operatorname{dim}_{\mathbb{R}}\left(g_{1} \mathfrak{t}_{I, 0} g_{1}^{-1}\right)=\operatorname{rank}\left(S_{1}\right)$ and thus $\rho\left(S_{2}\right)<\rho\left(S_{1}\right)$. Also recall that by Theorem 5.4 we have $\mathcal{T}(G) \cong \bigsqcup_{[I] \in \mathcal{E}_{W}} G / N_{G}\left(T_{I}\right)$. The map $\rho$ is constant on each connected component of the form $G / N_{G}\left(T_{I}\right)$. Thus $\rho: \mathcal{T}(G) \rightarrow \mathbb{N}$ is a continuous function. Finally, fix $0 \leq m \leq n$ and let $r$ be the rank of the center of $G$. Choose any set $I$ consisting of $m+r$ simple roots. Then the corresponding element $T_{I} \in \mathcal{T}(G)$ is such that $\rho\left(T_{I}\right)=m+r-r=m$.

Choose $n \geq 0$ as in the previous proposition and let $\mathcal{S}(n)$ be the poset consisting of all the nonempty subsets of $\{0,1, \ldots, n\}$, with the order given by the reverse inclusion of sets. We see an element in $\mathcal{S}(n)$ of the form $\boldsymbol{i}:=\left\{i_{0}, \ldots, i_{k}\right\}$, with $0 \leq i_{0}<i_{1}<\cdots<i_{k} \leq n$. Associated to the group $G$ we have a functor $\mathcal{F}_{G}: \mathcal{S}(n) \rightarrow$ Top defined in the following way. Suppose that $\boldsymbol{i}:=\left\{i_{0}, \ldots, i_{k}\right\}$ is an object in $\mathcal{S}(n)$. Then we define

$\mathcal{F}_{G}(\boldsymbol{i}):=\left\{\left(S_{0}, \ldots, S_{k}, a\right) \mid S_{0} \subset \cdots \subset S_{k} \in \mathcal{T}(G), \rho\left(S_{r}\right)=i_{r}\right.$ for $\left.0 \leq r \leq k, a \in B S_{0}\right\}$. 
Note that we have a natural inclusion $\mathcal{F}_{G}(\boldsymbol{i}) \subset \mathcal{T}(G)^{k+1} \times B G$ and we give $\mathcal{F}_{G}(\boldsymbol{i})$ the subspace topology. If $\boldsymbol{j}$ is a subset of $\boldsymbol{i}$ then the natural projection maps induce continuous functions $p_{\boldsymbol{i}, \boldsymbol{j}}: \mathcal{F}_{G}(\boldsymbol{i}) \rightarrow \mathcal{F}_{G}(\boldsymbol{j})$. This defines a functor $\mathcal{F}_{G}: \mathcal{S}(n) \rightarrow$ Top. To simplify matters, we use the following notation for elements in $\mathcal{F}_{G}(\boldsymbol{i})$. Given a chain $S_{0} \subset \cdots \subset S_{k}$ in $\mathcal{T}(G)$ with $\rho\left(S_{k}\right)=i_{r}$ for $0 \leq r \leq k$, we denote $S_{i}=\left(S_{0}, \ldots, S_{k}\right)$. With this notation the objects in $\mathcal{F}_{G}(i)$ are pairs of the form $\left(S_{i}, a\right)$ with $a \in B S_{0}$.

Theorem 6.3 Suppose that $G$ is a compact connected Lie group. Then there is a natural homotopy equivalence

$$
\underset{\boldsymbol{i} \in \mathcal{S}(n)}{\operatorname{hocolim}} \mathcal{F}_{G}(\boldsymbol{i}) \simeq B_{\mathrm{com}} G_{\mathbf{1}} .
$$

Proof The proof of this theorem will be divided into two steps. As a first step we construct a topological category $\mathcal{D}$ in such a way that there is a homotopy equivalence $B \mathcal{D} \simeq B_{\text {com }} G_{1}$.

Let $\mathcal{C}$ be the topological category whose objects are the elements in $B_{\text {com }} G_{\mathbf{1}}$ and the only morphisms are the identity maps. Since there are no nontrivial morphisms in $\mathcal{C}$ we have $B C=B_{\text {com }} G_{1}$ as topological spaces. Also, consider the topological category $\mathcal{D}$ defined as follows. The objects in $\mathcal{D}$ are pairs of the form $(S, a)$, where $S \in \mathcal{T}(G)$ and $a \in B S$. If $\left(S_{1}, a\right)$ and $\left(S_{2}, b\right)$ are two objects in $\mathcal{D}$, then there is a unique morphism $i:\left(S_{1}, a\right) \rightarrow\left(S_{2}, b\right)$ if and only if $a=b$ and $S_{1} \subset S_{2}$. We have a functor $F: \mathcal{D} \rightarrow \mathcal{C}$ that sends an object $(S, a)$ in $\mathcal{D}$ to $F(S, a)=a \in B S \subset B_{\text {com }} G_{\mathbf{1}}$. The functor $F$ sends every morphism in $\mathcal{D}$ to the corresponding identity morphism in $\mathcal{C}$. Fix an element $a \in B_{\mathrm{com}} G_{\mathbf{1}}$ and consider the under category $a \backslash F$. The objects in this category are tuples of the form $\left((S, a), \mathrm{id}_{a}\right)$, where $S \in \mathcal{T}(G)$ is such that $a \in B S$ and $\operatorname{id}_{a}: a \rightarrow a$ is the identity morphism in $\mathcal{C}$. There is a unique morphism $\left(\left(S_{1}, a\right), \mathrm{id}_{a}\right) \rightarrow\left(\left(S_{2}, a\right), \mathrm{id}_{a}\right)$ in $a \backslash F$ whenever $S_{1} \subset S_{2}$. We observe that the category $a \backslash F$ has an initial object. Indeed, let $S_{a}=\bigcap_{S \in \mathcal{T}(G), a \in B S} S$. Then $S_{a}$ is the smallest element in $\mathcal{T}(G)$ such that $a \in B S_{a}$ and this implies that $\left(\left(S_{a}, a\right), \mathrm{id}_{a}\right)$ is an initial object in $a \backslash F$. We conclude that the category $a \backslash F$ is a contractible category since it has an initial object. This means that is classifying space is contractible. Therefore as a particular case of $\left[17\right.$, Lemma A.5] we obtain that the map $B F: D \mathcal{D} \rightarrow B \mathcal{C} \cong B_{\text {com }} G_{\mathbf{1}}$ is a homotopy equivalence.

As a second step we show that there is a natural homeomorphism

$$
\phi: \underset{i \in \mathcal{S}(n)}{\operatorname{hocolim}} \mathcal{F}_{G}(\boldsymbol{i}) \rightarrow B \mathcal{D} .
$$

This will finish the proof. For this recall that by definition $\operatorname{hocolim}_{\boldsymbol{i} \in \mathcal{S}(n)} \mathcal{F}_{G}(\boldsymbol{i})=B \mathcal{G}$, where $\mathcal{G}$ is the topological category whose objects are sequences of the form $\left(\boldsymbol{i}, \boldsymbol{S}_{\boldsymbol{i}}, a\right)$. 
Here $\boldsymbol{i}=\left\{i_{0} \ldots, i_{k}\right\}$ is an element in $\mathcal{S}(n), \boldsymbol{S}_{\boldsymbol{i}}=\left(S_{0}, \ldots, S_{k}\right)$ is a chain in $\mathcal{T}(G)$ with $\rho\left(S_{r}\right)=i_{r}$ for $0 \leq r \leq k$ and $a \in B S_{0}$. Whenever $\boldsymbol{j} \subset \boldsymbol{i}$ there is a unique morphism in $\mathcal{G},\left(\boldsymbol{i}, \boldsymbol{S}_{\boldsymbol{i}}, a\right) \rightarrow\left(\boldsymbol{j}, \boldsymbol{S}_{\boldsymbol{j}}, a\right)$, which is induced by the corresponding projections. An element in $B \mathcal{G}$ is of the form $w=\left[\left(g_{1}, \ldots, g_{l}\right), t\right]$, where $t \in \Delta_{l}$ and $\left(g_{1}, \ldots, g_{l}\right)$ is a sequence of composable morphisms in $\mathcal{G}$ of the form

$$
\left(\boldsymbol{i}_{l}, \boldsymbol{S}_{\boldsymbol{i}_{l}}, a\right) \stackrel{g_{l}}{\longrightarrow}\left(\boldsymbol{i}_{l-1}, \boldsymbol{S}_{\boldsymbol{i}_{l-1}}, a\right) \stackrel{g_{l-1}}{\longrightarrow} \cdots \stackrel{g_{1}}{\longrightarrow}\left(\boldsymbol{i}_{0}, \boldsymbol{S}_{\boldsymbol{i}_{0}}, a\right) .
$$

This implies in particular that $\boldsymbol{i}_{0} \subset \cdots \subset \boldsymbol{i}_{l}$ is a nested sequence of nonempty sets. Write $\boldsymbol{i}:=\boldsymbol{i}_{l}=\left\{i_{0}, \ldots, i_{k}\right\}$ and $\boldsymbol{S}_{\boldsymbol{i}}=\left(S_{0}, \ldots, S_{k}\right)$. Therefore the morphisms $g_{1}, \ldots, g_{l}$ induce composable morphisms in $\mathcal{D}$

$$
\left(S_{0}, a\right) \stackrel{f_{1}}{\longrightarrow}\left(S_{1}, a\right) \stackrel{f_{2}}{\longrightarrow} \cdots \stackrel{f_{k}}{\longrightarrow}\left(S_{k}, a\right),
$$

where $f_{r}$ is the unique morphism in $\mathcal{D}$ from $\left(S_{r-1}, a\right)$ to $\left(S_{r}, a\right)$. Consider the standard $k$-simplex $\Delta_{k}$ that corresponds to the composable sequence $\left(f_{k}, \ldots, f_{1}\right)$ in $\mathcal{D}$. We identify the vertices of $\Delta_{k}$ with the numbers $i_{0}, \ldots, i_{k}$ by corresponding to each $i_{r}$ the vertex $v_{i_{r}}=(0, \ldots, 1, \ldots, 0) \in \Delta_{k}$, with the entry 1 in the $r^{\text {th }}$ position. If $\boldsymbol{j}=\left\{i_{r_{0}}, \ldots, i_{r_{m}}\right\} \subset \boldsymbol{i}$, we denote by $v_{\boldsymbol{j}}$ the barycenter of the simplex generated by the vertices $v_{i_{r_{0}}}, \ldots, v_{i_{r_{m}}}$. Thus with this notation, all the vertices in the barycentric subdivision of $\Delta_{k}, \mathcal{B} \Delta_{k}$, are of the form $v_{\boldsymbol{j}}$, where $\boldsymbol{j}$ is a nonempty subset of $\boldsymbol{i}$. We can associate to the nested sequence $\boldsymbol{i}_{0} \subset \cdots \subset \boldsymbol{i}_{l}$ the face of $\mathcal{B} \Delta_{k}$ generated by the vertices $v_{\boldsymbol{i}_{0}}, \ldots, v_{\boldsymbol{i}_{l}}$. Given $t=\left(t_{0}, \ldots, t_{l}\right) \in \Delta_{l}$, define $\gamma(t) \in \Delta_{k}$ by

$$
\gamma(t)=\gamma\left(t_{0}, \ldots, t_{l}\right)=t_{0} v_{\boldsymbol{i}_{l}}+i_{1} v_{\boldsymbol{i}_{l-1}}+\cdots+t_{l} v_{\boldsymbol{i}_{0}} .
$$

Using this convention we define

$$
\phi\left(\left[\left(g_{1}, \ldots, g_{l}\right), t\right]\right):=\left[\left(f_{k}, \ldots, f_{1}\right), \gamma(t)\right] \in B \mathcal{D} .
$$

In other words, the map $\phi$ is a linear isomorphism from the standard simplex $\Delta_{l}$, that corresponds to the composable sequence $\left(g_{1}, \ldots, g_{l}\right)$ in $\mathcal{G}$, onto the face of $\mathcal{B} \Delta_{k}$ that corresponds to the chain $\boldsymbol{i}_{0} \subset \boldsymbol{i}_{2} \subset \cdots \subset \boldsymbol{i}_{l}$, where $\Delta_{k}$ is the simplex associated to the composable sequence $\left(i_{k}, \ldots, i_{1}\right)$ in $\mathcal{D}$. It can be seen that the map $\phi$ is well defined and is in fact a homeomorphism.

The values of the functor $\mathcal{F}_{G}$ can be described explicitly as follows. Fix $\boldsymbol{i}=\left\{i_{0}, \ldots, i_{k}\right\}$ an element in $\mathcal{S}(n)$. The conjugation action of $G$ defines an equivalence relation on the set of chains in $\mathcal{T}(G)$ in the following way. Suppose that $S_{i}=\left\{S_{0} \subset \cdots \subset S_{k}\right\}$ and $S_{i}^{\prime}=\left\{S_{0}^{\prime} \subset \cdots \subset S_{k}^{\prime}\right\}$ are two chains with $\rho\left(S_{r}\right)=\rho\left(S_{r}^{\prime}\right)=i_{r}$ for $0 \leq r \leq k$. Then we say that $\boldsymbol{S}_{\boldsymbol{i}} \sim \boldsymbol{S}_{\boldsymbol{i}}^{\prime}$ if and only if we can find some $g \in G$ such that $\boldsymbol{S}_{\boldsymbol{i}}=g \boldsymbol{S}_{\boldsymbol{i}}^{\prime} g^{-1}$; that is, $S_{r}=g S_{r}^{\prime} g^{-1}$ for all $0 \leq r \leq k$. Denote by $\mathcal{E}(\boldsymbol{i})$ the set of all equivalence 
classes of such chains and by $\left[S_{i}\right]$ the equivalence class representing $S_{i}$ in $\mathcal{E}(i)$. Fix a chain $S_{i}:=\left\{S_{0} \subset \cdots \subset S_{k}\right\}$ in $\mathcal{T}(G)$ with $\rho\left(S_{r}\right)=i_{r}$ for $0 \leq r \leq k$. The conjugation action of $G$ induces a continuous map

$$
\bar{\mu}_{S_{i}}: G \times B S_{0} \rightarrow \mathcal{F}_{G}(i), \quad(g, a) \mapsto\left(g S_{i} g^{-1}, g a g^{-1}\right) .
$$

Let $N_{G}\left(S_{i}\right)$ be the normalizer of $S_{i}$ in $G$; that is, the subgroup of $G$ consisting of elements $g \in G$ such that $g S_{i} g^{-1}=S_{i}$. The group $N_{G}\left(S_{i}\right)$ acts by conjugation on $B S_{0}$ and on the left on $G$ by the assignment $n \cdot g=g n^{-1}$. This induces a diagonal action of $N_{G}\left(\boldsymbol{S}_{i}\right)$ on $G \times B S_{0}$ and the map $\bar{\mu}_{\boldsymbol{S}_{\boldsymbol{i}}}$ is invariant under this action. Therefore $\bar{\mu}_{\boldsymbol{S}_{\boldsymbol{i}}}$ induces a continuous function

$$
\mu_{\boldsymbol{S}_{\boldsymbol{i}}}: G \times_{N_{G}\left(\boldsymbol{S}_{\boldsymbol{i}}\right)} B S_{0} \rightarrow \mathcal{B}_{\mathcal{T}(G)}(\boldsymbol{i}) .
$$

If we vary $S_{\boldsymbol{i}}$ through the different equivalence classes in $\mathcal{E}(\boldsymbol{i})$, then we obtain a continuous map

$$
\mu_{\boldsymbol{i}}=\bigsqcup_{\left[\boldsymbol{S}_{\boldsymbol{i}}\right] \in \mathcal{E}(\boldsymbol{i})} \mu_{\boldsymbol{S}_{\boldsymbol{i}}}: \bigsqcup_{\left[\boldsymbol{S}_{\boldsymbol{i}}\right] \in \mathcal{E}(\boldsymbol{i})} G \times_{N_{G}\left(\boldsymbol{S}_{\boldsymbol{i}}\right)} B S_{0} \rightarrow \mathcal{F}_{G}(\boldsymbol{i}) .
$$

This map is clearly surjective. In fact this map is also injective. Indeed, suppose that

$$
\mu_{\boldsymbol{i}}(g, a)=\left(g \boldsymbol{S}_{\boldsymbol{i}} g^{-1}, g a g^{-1}\right)=\left(g_{1} \boldsymbol{S}_{\boldsymbol{i}}^{\prime} g_{1}^{-1}, g_{1} a_{1} g_{1}^{-1}\right)=\mu_{\boldsymbol{i}}\left(g_{1}, a_{1}\right) .
$$

Then $g \boldsymbol{S}_{\boldsymbol{i}} g^{-1}=g_{1} \boldsymbol{S}_{\boldsymbol{i}}^{\prime} g_{1}^{-1}$, which means that $\left[\boldsymbol{S}_{\boldsymbol{i}}\right]=\left[\boldsymbol{S}_{\boldsymbol{i}}^{\prime}\right]$. Thus we can assume without loss of generality that $S_{i}=S_{i}^{\prime}$. Also we have $g_{1}^{-1} g S_{i}\left(g_{1}^{-1} g\right)^{-1}=S_{i}$ and $\mathrm{gag}^{-1}=g_{1} a_{1} g_{1}^{-1}$. Therefore $n:=g_{1}^{-1} g \in N_{G}\left(\boldsymbol{S}_{\boldsymbol{i}}\right)$ is such that $\mathrm{nan}^{-1}=a_{1}$. We conclude that in $G \times \times_{N_{G}}\left(\boldsymbol{S}_{i}\right) B S_{0}$ we have $[(g, a)]=\left[g n^{-1}, n a n^{-1}\right]=\left[\left(g_{1}, a_{1}\right)\right]$, proving that $\mu_{\boldsymbol{i}}$ is injective. Moreover, it can easily be seen that $\mu_{\boldsymbol{i}}^{-1}$ is also continuous and thus $\mu_{\boldsymbol{i}}$ is a homeomorphism. We conclude that for every element $\boldsymbol{i}$ in $\mathcal{S}(n)$ there is a natural homeomorphism

$$
\mathcal{F}_{G}(\boldsymbol{i}) \cong \bigsqcup_{\left[S_{i}\right] \in \mathcal{E}(\boldsymbol{i})} G \times_{N_{G}\left(S_{i}\right)} B S_{0} .
$$

The sets $\mathcal{E}(\boldsymbol{i})$ that appear in the previous description can be expressed in terms of the root system $\Phi$ associated to a maximal torus $T \subset G$ in the following way. Let $\boldsymbol{i}=\left\{i_{0}, \ldots, i_{k}\right\}$ be an object in $\mathcal{S}(n)$ and $S_{\boldsymbol{i}}=\left\{S_{0} \subset \cdots \subset S_{k}\right\}$ a chain in $\mathcal{T}(G)$ with $\rho\left(S_{r}\right)=i_{r}$ for $0 \leq r \leq k$. After replacing $S_{\boldsymbol{i}}$ with a suitable conjugate we may assume that $S_{\boldsymbol{i}}$ is such that $S_{0} \subset \cdots \subset S_{k} \subset T$. By Theorem 5.4, for every $0 \leq r \leq k$ we can find a set of simple roots $I_{r}$ in such a way that $S_{r}=g_{r} T_{I_{r}} g_{r}^{-1}$ for some $g_{r} \in G$. Let $T_{I_{r}, 0}$ denote the connected component of $T_{I_{r}}$ that contains the identity. Then $T_{I_{r}, 0}$ is a torus since it is a compact, connected abelian Lie group. Therefore for each $0 \leq r \leq k$ we can find some element $x_{r}$ such that $T_{I_{r}, 0}=\overline{\left\langle x_{r}\right\rangle}$. Each $x_{r}$ 
is such that $x_{r} \in T_{I_{r}} \subset T$ and also $g_{r} x_{r} g_{r}^{-1} \in T$. By [10, Lemma IV 2.5], for each $1 \leq r \leq k$ we can find some $w_{r} \in W$ such that $w_{r} x_{r}=g_{r} x_{r} g_{r}^{-1}$. We conclude then that $S_{r, 0}=w_{r} T_{I_{r}, 0}$ and this in turn implies that $S_{r}=w_{r} T_{I_{r}}=T_{w_{r}^{-1} I_{r}}$ for all $1 \leq r \leq k$. This proves that any chain $S_{i}=\left\{S_{0} \subset \cdots \subset S_{k}\right\}$ in $\mathcal{T}(G)$ is conjugated to a chain of the form $T_{J_{0}} \subset T_{J_{1}} \subset \cdots \subset T_{J_{k}}$ for a collection of sets of roots $J_{0}, \ldots, J_{k}$. Moreover, two chains such chains $T_{I_{0}} \subset T_{I_{1}} \subset \cdots \subset T_{I_{k}}$ and $T_{J_{0}} \subset T_{J_{1}} \subset \cdots \subset T_{J_{k}}$ are conjugated if and only if we can find some $w \in W$ such that $T_{I_{r}}=T_{w J_{r}}$ for $0 \leq r \leq k$. This proves that the set $\mathcal{E}(\boldsymbol{i})$ can be identified with the set of equivalence classes of sequences of sets of roots of the form $\left(J_{0}, \ldots, J_{k}\right)$ with $\rho\left(T_{J_{r}}\right)=i_{r}$ for $0 \leq r \leq k$, where $\left(J_{0}, \ldots, J_{k}\right) \sim\left(I_{0}, \ldots, I_{k}\right)$ if and only if we can find some $w \in W$ such that $T_{I_{r}}=T_{w J_{r}}$ for $0 \leq r \leq k$.

Example 6.4 Take $G=\mathrm{SU}(2)$, which is a Lie group of rank 1. In this case the poset $\mathcal{T}(G)$ has one element corresponding to the center of $G$ (isomorphic to $\mathbb{Z} / 2$ ) which has rank zero and an element for every maximal torus in $G$. Fix $T \subset G$ the maximal torus consisting of those $2 \times 2$ diagonal matrices in $G$. The Weyl group $W=\mathbb{Z} / 2$ acts by permutation on the diagonal entries for such matrices. The space of all maximal tori in $G$ is homeomorphic to $G / N_{G}(T)$. Therefore $\mathcal{T}(G)=* \sqcup G / N_{G}(T) \cong * \sqcup \mathbb{R} \mathrm{P}^{2}$. By Theorem 6.3 it follows that $B_{\text {com }} G \simeq \operatorname{hocolim}_{\boldsymbol{i} \in \mathcal{S}(1)} \mathcal{F}_{G}(\boldsymbol{i})$. In this case we have

$$
\begin{aligned}
\mathcal{F}_{G}(0) & =B Z(G)=B \mathbb{Z} / 2=\mathbb{R} \mathrm{P}^{\infty}, \\
\mathcal{F}_{G}(1) & =G / T \times_{W} B T=\mathbb{S}^{2} \times_{\mathbb{Z} / 2} \mathbb{C P}^{\infty}, \\
\mathcal{F}_{G}(0,1) & =G / N_{G}(T) \times B \mathbb{Z} / 2=\mathbb{R} \mathrm{P}^{2} \times \mathbb{R} \mathrm{P}^{\infty} .
\end{aligned}
$$

Therefore $B_{\text {com }} G$ is homotopy equivalent to the homotopy pushout of the diagram

$$
\mathcal{F}_{G}(0) \stackrel{p_{0}}{\longleftarrow} \mathcal{F}_{G}(0,1) \stackrel{p_{1}}{\longrightarrow} \mathcal{F}_{G}(1),
$$

where

$$
p_{0}: \mathcal{F}_{G}(0,1) \cong \mathbb{R} \mathrm{P}^{2} \times \mathbb{R} \mathrm{P}^{\infty} \rightarrow \mathcal{F}_{G}(0) \cong \mathbb{R} \mathrm{P}^{\infty}
$$

corresponds to second projection and

$$
p_{1}: \mathcal{F}_{G}(0,1) \cong \mathbb{R} \mathrm{P}^{2} \times \mathbb{R} \mathrm{P}^{\infty} \rightarrow \mathcal{F}_{G}(1) \cong \mathbb{S}^{2} \times_{\mathbb{Z} / 2} \mathbb{C P} \mathrm{P}^{\infty}
$$

is the map induced by the inclusion $\mathbb{Z} / 2 \hookrightarrow T$. Using the associated Mayer-Vietoris sequence we obtain

$$
H^{k}\left(B_{\mathrm{com}} \mathrm{SU}(2) ; \mathbb{Z}\right) \cong \begin{cases}\mathbb{Z} & \text { if } k=0 \\ 0 & \text { if } k=2 \text { or } k \text { odd }, \\ \mathbb{Z} \oplus \mathbb{Z} & \text { if } k \equiv 0(\bmod 4), k>0 \\ \mathbb{Z} / 2 & \text { if } k \equiv 2(\bmod 4), k>2\end{cases}
$$


Note in particular the presence of a $\mathbb{Z} / 2$-factor in cohomological degrees $k \equiv 2(\bmod 4)$ and $k>2$. The existence of this $2-$ torsion is particularly intriguing and we are interested in finding a suitable geometric interpretation.

Suppose now that $G$ is a Lie group that is compact and connected Lie group with center $Z$. Let $n=\operatorname{rank}(G)-\operatorname{rank}(Z)$. Define a functor $\mathcal{H}_{G}: \mathcal{S}(n) \rightarrow$ Top as follows. If $\boldsymbol{i}=\left\{i_{0}, \ldots, i_{k}\right\}$ is an object in $\mathcal{S}(n)$ then we define

$\mathcal{H}_{G}(\boldsymbol{i}):=\left\{\left(S_{0}, \ldots, S_{k}, x\right) \mid S_{0} \subset \cdots \subset S_{k} \in \mathcal{T}(G), \rho\left(S_{r}\right)=i_{r}\right.$ for $\left.0 \leq r \leq k, x \in G / S_{0}\right\}$.

If $\boldsymbol{j}$ is a subset of $\boldsymbol{i}$ then the corresponding function is the map $p_{\boldsymbol{i}, \boldsymbol{j}}: \mathcal{H}_{G}(\boldsymbol{i}) \rightarrow \mathcal{H}_{G}(\boldsymbol{j})$ induced by the projection maps and the quotient map.

Theorem 6.5 Suppose that $G$ is a compact connected Lie group. Then there is a natural $G$-equivariant homotopy equivalence

$$
\underset{\boldsymbol{i} \in \mathcal{S}(n)}{\operatorname{hocolim}} \mathcal{H}_{G}(\boldsymbol{i}) \simeq E_{\mathrm{com}} G_{\mathbf{1}} .
$$

Proof Define a functor $\widetilde{\mathcal{H}}_{G}: \mathcal{S}(n) \rightarrow$ Top that associates an object $\boldsymbol{i}$ in $\mathcal{S}(n)$ with $\widetilde{\mathcal{H}}_{G}(\boldsymbol{i}):=\left\{\left(S_{0}, \ldots, S_{k}, x\right) \mid S_{0} \subset \cdots \subset S_{k}, \rho\left(S_{r}\right)=i_{r}\right.$ for $\left.0 \leq r \leq k, x \in p_{\text {com }}^{-1}\left(B S_{0}\right)\right\}$, where $p_{\mathrm{com}}: E_{\mathrm{com}} G_{\mathbf{1}} \rightarrow B_{\mathrm{com}} G$ is the projection map. Note that $p_{\mathrm{com}}^{-1}\left(B S_{0}\right)$ is the geometric realization of the subsimplicial space of $\left[E_{\mathrm{com}} G\right]_{*}$ whose $n^{\text {th }}$ space is $\operatorname{Hom}\left(\mathbb{Z}^{n}, S_{0}\right) \times G=S_{0}^{n} \times G$. In particular we have a homotopy equivalence $p_{\text {com }}^{-1}\left(B S_{0}\right) \simeq E S_{0} \times S_{0} G \simeq G / S_{0}$. Using this equivalence we obtain a natural transformation $\mu: \mathcal{H}_{G} \rightarrow \widetilde{\mathcal{H}}_{G}$ such that $\mu_{\boldsymbol{i}}: \mathcal{H}_{G}(\boldsymbol{i}) \rightarrow \widetilde{\mathcal{H}}_{G}(\boldsymbol{i})$ is a $G$-equivariant homotopy equivalence for every $\boldsymbol{i}$. We conclude then that there is a $G$-equivariant homotopy equivalence

$$
\underset{\boldsymbol{i} \in \mathcal{S}(n)}{\operatorname{hocolim}} \mathcal{H}_{G}(\boldsymbol{i}) \simeq \underset{\boldsymbol{i} \in \mathcal{S}(n)}{\operatorname{hocolim}} \tilde{\mathcal{H}}_{G}(\boldsymbol{i}) .
$$

On the other hand, let $\mathcal{D}$ be the topological category whose objects are pairs of the form $(S, x)$, where $S \in \mathcal{T}(G)$ and $x \in p_{\text {com }}^{-1}(B S)$. There is a unique morphism $\left(S_{1}, x\right) \rightarrow\left(S_{2}, y\right)$ in $\mathcal{D}$ if and only if $x=y$ and $S_{1} \subset S_{2}$. An argument similar to the one provided in Theorem 6.3 shows that there is a $G$-equivariant homeomorphism hocolim $_{\boldsymbol{i} \in \mathcal{S}(n)} \mathcal{H}_{G}(\boldsymbol{i}) \cong B \mathcal{D}$. Finally, let $\mathcal{C}$ be the topological category whose objects are the elements in $E_{\text {com }} G_{1}$ and the only morphisms are the identity morphisms. Thus we have $B \mathcal{C}=E_{\text {com }} G_{\mathbf{1}}$. Let $F: \mathcal{D} \rightarrow \mathcal{C}$ be the functor that for an object $(S, x)$ in $\mathcal{D}$ corresponds $F(S, x)=x \in p_{\text {com }}^{-1}(B S) \subset E_{\text {com }} G_{\mathbf{1}}$. The functor $F$ sends any morphism in $\mathcal{D}$ to the corresponding identity morphism in $\mathcal{C}$. Using the same argument as in 
Theorem 6.3 we conclude that the map $B F: B \mathcal{D} \rightarrow B C$ is a $G$-equivariant homotopy equivalence.

Remark 6.6 Let $Y(G):=\operatorname{hocolim}_{\boldsymbol{i} \in \mathcal{S}(n)} \mathcal{H}_{G}(\boldsymbol{i})$. Note that $Y(G)$ is a finite $G-\mathrm{CW}-$ complex and by Theorem 6.5 we have $B_{\text {com }} G_{1} \simeq E G \times_{G} Y(G)$. When $G$ is abelian $Y(G)$ is a contractible space, and so it can be seen as measuring how far $G$ is from being a commutative group. Using the Atiyah-Segal completion theorem we conclude that $K^{*}\left(B_{\text {com }} G_{1}\right)$ is the completion of $K_{G}^{*}(Y(G))$ with respect to the augmentation ideal $I_{G}$ in the complex representation ring $R(G)$ of $G$. This can be seen as a generalization of the classical computation $K^{*}(B G) \cong R(G)_{I_{G}}^{\wedge}$ for a compact Lie group $G$.

\section{Rational cohomology of $B_{\operatorname{com}} G_{1}$}

In this section we provide computations for the cohomology of the spaces $B_{\text {com }} G_{\mathbf{1}}$ with rational coefficients for a real or complex reductive algebraic group $G$ that is connected as a topological space. By Theorem 3.1 we can work with compact connected Lie groups without loss of generality. Throughout this section we take the rational numbers as the ground field for all computations unless otherwise specified.

Fix a compact connected Lie group $G$. Let $T \subset G$ be a maximal torus and let $W$ be the corresponding Weyl group. For every $n \geq 0$ consider the map

$$
\begin{aligned}
\bar{\varphi}_{n}: G \times T^{n} & \rightarrow \operatorname{Hom}\left(\mathbb{Z}^{n}, G\right)_{\mathbf{1}}, \\
\left(g, t_{1}, \ldots, t_{n}\right) & \mapsto\left(g t_{1} g^{-1}, \ldots, g t_{n} g^{-1}\right) .
\end{aligned}
$$

The group $N_{G}(T)$ acts naturally on $G \times T^{n}$ by

$$
n \cdot\left(g, t_{1}, \ldots, t_{n}\right):=\left(g n^{-1}, n t_{1} n^{-1}, \ldots, n t_{n} n^{-1}\right) .
$$

The map $\bar{\varphi}_{n}$ is invariant under this action; as a result we obtain a continuous map

$$
\begin{aligned}
\varphi_{n}: G / T \times_{W} T^{n}=G \times_{N_{G}(T)} T^{n} & \rightarrow \operatorname{Hom}\left(\mathbb{Z}^{n}, G\right) \mathbf{1}, \\
{\left[\left(g, t_{1}, \ldots, t_{n}\right)\right] } & \mapsto\left(g t_{1} g^{-1}, \ldots, g t_{n} g^{-1}\right) .
\end{aligned}
$$

Here $W$ acts diagonally on $T^{n}$. This map is surjective as any $n$-tuple $\left(g_{1}, \ldots, g_{n}\right)$ of elements in $G$ belongs to $\operatorname{Hom}\left(\mathbb{Z}^{n}, G\right)_{1}$ if and only if there is a maximal torus in $G$ that contains $g_{1}, \ldots, g_{n}$ and all maximal tori in $G$ are conjugated. By [8, Lemma 3.2] it follows that the fibers of $\varphi_{n}$ are rationally acyclic and thus $\varphi_{n}$ induces an isomorphism in cohomology with rational coefficients. It is easy to see that the collection $\left\{\varphi_{n}\right\}_{n \geq 0}$ defines a map of simplicial spaces and by passing to the geometric realization we obtain a continuous surjective map

$$
\varphi: G / T \times_{W} B T \rightarrow B_{\mathrm{com}} G_{\mathbf{1}} .
$$


In the same way as in [3, Theorem 6.1], we conclude that the map $\varphi$ induces an isomorphism in cohomology with rational coefficients and thus we obtain an isomorphism

$$
\varphi^{*}: H^{*}\left(B_{\mathrm{com}} G_{\mathbf{1}}\right) \stackrel{\cong}{\rightarrow}\left(H^{*}(G / T) \otimes H^{*}(B T)\right)^{W},
$$

with $W$ acting diagonally on $H^{*}(G / T) \otimes H^{*}(B T)$. This can be used to provide the following useful identification of the rational cohomology of $B_{\mathrm{com}} G_{\mathbf{1}}$.

Proposition 7.1 Suppose that $G$ is a compact connected Lie group and let $T \subset G$ be a maximal torus. Then there is a natural isomorphism of rings

$$
\alpha_{G}: H^{*}\left(B_{\mathrm{com}} G_{\mathbf{1}}\right) \stackrel{\cong}{\rightrightarrows}\left(H^{*}(B T) \otimes H^{*}(B T)\right)^{W} / J_{G} .
$$

In the above equation $W$ acts diagonally on $H^{*}(B T) \otimes H^{*}(B T)$ and $J_{G}$ is the ideal generated by the elements of positive degrees in the image of

$$
\begin{aligned}
i_{1}: H^{*}(B G) & \rightarrow\left(H^{*}(B T) \otimes H^{*}(B T)\right)^{W}, \\
x & \mapsto x \otimes 1 .
\end{aligned}
$$

Proof The Eilenberg-Moore spectral sequence with $\mathbb{Q}$-coefficients associated to the fibration

$$
G / T \rightarrow B T \stackrel{i}{\rightarrow} B G
$$

collapses at the $E_{2}$-term (see [20, page 278]). Also, there is a $W$-equivariant isomorphism of graded rings $H^{*}(G / T) \cong H^{*}(B T) / I_{G}$, where $I_{G}$ is the ideal in $H^{*}(B T)$ generated by the elements of positive degree in the image of $i^{*}: H^{*}(B G) \rightarrow H^{*}(B T)$. Consider now the natural map

$$
\pi: H^{*}(B T) \otimes H^{*}(B T) \rightarrow\left(H^{*}(B T) / I_{G}\right) \otimes H^{*}(B T) .
$$

This is a surjective map whose kernel is the ideal $\widetilde{I}_{G}$ generated by the elements of positive degree in the image of the map $i_{1}: H^{*}(B G) \rightarrow H^{*}(B T) \otimes H^{*}(B T)$ given by $x \mapsto x \otimes 1$. Thus we have a short exact sequence

$$
0 \rightarrow \tilde{I}_{G} \rightarrow H^{*}(B T) \otimes H^{*}(B T) \rightarrow\left(H^{*}(B T) / I_{G}\right) \otimes H^{*}(B T) \rightarrow 0 .
$$

Since we are working in characteristic zero and $W$ is a finite group, the exactness of this sequence is preserved at the level of $W$-invariants; that is, there is a short exact sequence

$(7-2) 0 \rightarrow \widetilde{I}_{G}^{W} \rightarrow\left(H^{*}(B T) \otimes H^{*}(B T)\right)^{W} \rightarrow\left(\left(H^{*}(B T) / I_{G}\right) \otimes H^{*}(B T)\right)^{W} \rightarrow 0$.

Note that $J_{G}=\widetilde{I}_{G}^{W}$; thus we obtain a natural isomorphism

$$
\psi:\left(H^{*}(B T) \otimes H^{*}(B T)\right)^{W} / J_{G} \rightarrow\left(H^{*}(G / T) \otimes H^{*}(B T)\right)^{W} .
$$

The required isomorphism is then $\alpha_{G}:=\psi^{-1} \circ \varphi$, where $\varphi$ is as in (7-1). 
The previous proposition has a number of interesting applications. To start note that we have a natural inclusion $B T \subset B_{\text {com }} G_{1} \subset B G$. At the level of cohomology groups this induces a natural monomorphism $H^{*}(B G) \hookrightarrow H^{*}\left(B_{\text {com }} G_{\mathbf{1}}\right)$ and thus we can consider $H^{*}\left(B_{\text {com }} G_{1}\right)$ as a module over $H^{*}(B G)$. Under the identification

$$
\alpha: H^{*}\left(B_{\mathrm{com}} G_{\mathbf{1}}\right) \stackrel{\cong}{\rightrightarrows}\left(H^{*}(B T) \otimes H^{*}(B T)\right)^{W} / J_{G}
$$

provided in the previous proposition, this structure as $H^{*}(B G)$-module corresponds to the structure on $\left(H^{*}(B T) \otimes H^{*}(B T)\right)^{W} / J_{G}$ given by $g \cdot[x \otimes y]:=[x \otimes g y]$. As a consequence of this we derive the following theorem.

Theorem 7.2 Suppose that $G$ is a compact, connected Lie group. Then

$$
H^{*}\left(B_{\mathrm{com}} G_{\mathbf{1}}\right)
$$

is a free module over $H^{*}(B G)$ of rank $|W|$, where $W$ is the corresponding Weyl group.

Proof Fix a maximal torus $T \subset G$ and let $W$ be the corresponding Weyl group. Let $S=H^{*}(B G)$ and $A=H^{*}(B T)$. These are graded rings, $W$ acts on $A$ with degree-preserving ring automorphisms and we have a natural isomorphism $S \cong A^{W}$. The ring $S$ is a polynomial ring in finitely many commuting variables. Also, the ring $A$ can be seen as a graded module over $S$ and this is in fact a free module of rank $|W|$. Consider $M^{W}:=(A \otimes A)^{W}$. This is a graded ring that contains $R:=S \otimes S$ as a subring. Thus $M^{W}$ can be seen as a graded module over $R$. As a first step we will show that $M^{W}$ is a finitely generated free $R$-module. To this end, note that $R$ is a Cohen-Macaulay ring as it is a polynomial ring over $\mathbb{Q}$. The same is true for $A \otimes A$. Since $W$ acts by degree-preserving ring automorphisms on $A \otimes A$, it follows that $M^{W}=(A \otimes A)^{W}$ is also a Cohen-Macaulay ring by [15, Proposition 13]. We observe that $M^{W}$ is finitely generated as an $R$-module. Indeed, suppose $\left\{e_{w}\right\}_{w \in W}$ is a free basis of $A$ as a module over $S$. Then $\left\{e_{v} \otimes e_{w}\right\}_{v, w \in W}$ is a free basis of $A \otimes A$ as a module over $R=S \otimes S$. Define the averaging operator

$$
\begin{aligned}
\rho: A \otimes A & \rightarrow(A \otimes A)^{W}=M^{W}, \\
f & \mapsto \frac{1}{|W|} \sum_{w \in W} w \cdot f .
\end{aligned}
$$

The map $\rho$ is surjective and $R$-linear; this implies that the collection $\left\{\rho\left(e_{v} \otimes e_{w}\right)\right\}_{v, w \in W}$ generates $M^{W}$ as a module over $R$. Thus $M^{W}$ is a finitely generated $R$-module. Since $R$ is a polynomial algebra over $\mathbb{Q}$, then any finitely generated $R$-module has finite projective dimension. Using the Auslander-Buchsbaum formula for graded rings we obtain

$$
\operatorname{pd}_{R}\left(M^{W}\right)=\operatorname{depth}(R)-\operatorname{depth}\left(M^{W}\right),
$$


where $\operatorname{pd}_{R}\left(M^{W}\right)$ is the projective dimension of $M^{W}$ as an $R$-module. Since both $M^{W}$ and $R$ are Cohen-Macaulay rings and $M^{W}$ is finitely generated as an $R$-module, this implies $\operatorname{pd}_{R}\left(M^{W}\right)=\operatorname{dim}(R)-\operatorname{dim}\left(M^{W}\right)=0$. This means that $M^{W}$ is projective as an $R$-module and by the Quillen-Suslin theorem (see [24, Theorem 4]), it follows that $M^{W}$ is a free $R$-module. Recall that $J_{G}$ is the ideal in $M^{W}$ generated by the elements of positive degree of the form $x \otimes 1$ for $x \in S$. Suppose that $\left\{a_{w}\right\}_{w}$ is a free basis of $M^{W}$ as a module over $R$. If $f_{w}=\bar{a}_{w}$ is the image of $a_{w}$ in $M^{W} / J_{G}$ under the natural map, then it follows that $\left\{f_{w}\right\}_{w}$ is a free basis of $M^{W} / J_{G}$ as a module over $S$. By Proposition 7.1 this means that $H^{*}\left(B_{\mathrm{com}} G_{1}\right)$ is free as a module over $H^{*}(B G)$. To finish we only need to compute the rank of $H^{*}\left(B_{\mathrm{com}} G_{\mathbf{1}}\right)$. For this recall that we have a natural isomorphism $\varphi^{*}: H^{*}\left(B_{\text {com }} G_{1}\right) \stackrel{\cong}{\rightrightarrows}\left(H^{*}(G / T) \otimes H^{*}(B T)\right)^{W}$. As an ungraded $W$-module $H^{*}(G / T)$ is isomorphic to the regular $W$-representation. It follows that as an ungraded module, $H^{*}\left(B_{\text {com }} G_{1}\right)$ is isomorphic to $H^{*}(B T)$ and the latter has rank $|W|$ as a module over $H^{*}(B G)$. This implies that as a graded $H^{*}(B G)$-module $H^{*}\left(B_{\text {com }} G_{\mathbf{1}}\right)$ is free and of rank $|W|$.

Remark 7.3 The previous theorem is not true in general if we use integer coefficients. For example if $G=\mathrm{SU}(2)$ then $H^{*}\left(B_{\text {com }} \mathrm{SU}(2) ; \mathbb{Z}\right)$ is not free as a module over $H^{*}(B \mathrm{SU}(2) ; \mathbb{Z})$ because the former contains 2-torsion as we proved in Example 6.4 and the latter does not contain torsion.

Consider now the inclusion map $i: B_{\text {com }} G_{\mathbf{1}} \rightarrow B G$. Up to homotopy we have a fibration sequence

$$
E_{\mathrm{com}} G_{\mathbf{1}} \stackrel{p_{\mathrm{com}}}{\longrightarrow} B_{\mathrm{com}} G_{\mathbf{1}} \stackrel{i}{\rightarrow} B G .
$$

Since $G$ is assumed to be connected then the base space, $B G$, is simply connected. The $E_{2}$-term of the Eilenberg-Moore spectral sequence with $\mathbb{Q}$-coefficients associated to the fibration (7-3) is

$$
E_{2}^{*, *}=\operatorname{Tor}_{H^{*}(B G)}\left(\mathbb{Q}, H^{*}\left(B_{\mathrm{com}} G_{\mathbf{1}}\right)\right)
$$

and this spectral sequence converges strongly to $H^{*}\left(E_{\mathrm{com}} G_{\mathbf{1}}\right)$. By the previous theorem, if $G$ is a compact connected Lie group then $H^{*}\left(B_{\text {com }} G_{1}\right)$ is a free module over $H^{*}(B G)$. It follows that

$$
\operatorname{Tor}_{H^{*}(B G)}\left(\mathbb{Q}, H^{*}\left(B_{\mathrm{com}} G_{\mathbf{1}}\right)\right) \cong \mathbb{Q} \otimes_{H^{*}(B G)} H^{*}\left(B_{\mathrm{com}} G_{\mathbf{1}}\right)
$$

and the Eilenberg-Moore spectral sequence collapses to the $E_{2}^{0, *}-$ column. The map $p_{\mathrm{com}}^{*}: H^{*}\left(B_{\mathrm{com}} G_{\mathbf{1}}\right) \rightarrow H^{*}\left(E_{\mathrm{com}} G_{\mathbf{1}}\right)$ is surjective since $\operatorname{Im}\left(p_{\mathrm{com}}^{*}\right)=E_{\infty}^{0, *}$ and the sequence collapses at the $E_{2}^{0, *}-$ column. Let $K_{G}$ denote the ideal in $H^{*}\left(B_{\text {com }} G_{1}\right)$ generated by the elements in $H^{*}(B G)$ of positive degree. Then $\operatorname{Ker}\left(p_{\text {com }}^{*}\right)=K_{G}$ and 
we conclude that there is an isomorphism of rings $H^{*}\left(E_{\text {com }} G_{\mathbf{1}}\right) \cong H^{*}\left(B_{\text {com }} G_{\mathbf{1}}\right) / K_{G}$. Using the isomorphism provided in Proposition 7.1 we conclude that if $L_{G}$ is the ideal in $\left(H^{*}(B T) \otimes H^{*}(B T)\right)^{W}$ generated by the elements of positive degree in the image of $H^{*}(B G) \otimes H^{*}(B G)$, then there is a natural isomorphism $H^{*}\left(E_{\text {com }} G_{\mathbf{1}}\right) \cong$ $\left(H^{*}(B T) \otimes H^{*}(B T)\right)^{W} / L_{G} \cong\left(H^{*}(G / T) \otimes H^{*}(G / T)\right)^{W}$. This proves the following corollary.

Corollary 7.4 Suppose that $G$ is a connected compact Lie group with maximal torus $T$ and associated Weyl group $W$. Then there is a natural isomorphism of rings

$$
H^{*}\left(E_{\mathrm{com}} G_{\mathbf{1}}\right) \cong\left(H^{*}(G / T) \otimes H^{*}(G / T)\right)^{W},
$$

and the Poincaré series of $B_{\mathrm{com}} G_{1}$ and $E_{\mathrm{com}} G_{1}$ satisfy

$$
P_{B_{\mathrm{com}} G_{\mathbf{1}}}(t)=P_{B G}(t) P_{E_{\mathrm{com}} G_{\mathbf{1}}}(t) .
$$

Note that $G / T \times G / T$ is a compact, orientable manifold and that $W$ preserves orientation. Hence we infer that the fundamental class is $W$-invariant. This yields the following:

Corollary 7.5 These statements are equivalent for a compact connected Lie group $G$ :

(1) $E_{\mathrm{com}} G_{\mathbf{1}}$ is contractible.

(2) $E_{\mathrm{com}} G_{\mathbf{1}}$ is rationally acyclic.

(3) $G$ is abelian.

Proof If $E_{\mathrm{com}} G_{\mathbf{1}}$ is contractible then it is acyclic. If it is acyclic, then $G / T$ must be zero-dimensional, hence $G=T$ and so $G$ is abelian. If $G$ is abelian $B_{\text {com }} G_{\mathbf{1}}=B G$ and so $E_{\text {com }} G_{\mathbf{1}}$ is contractible.

From the description given in Corollary 7.4, it follows that the Poincare series for $E_{\text {com }} G_{1}$ encodes information about all the complex irreducible representations of the Weyl group $W$ associated to the pair $(G, T)$. To see this recall that as an ungraded $W$-representation $H^{*}(G / T ; \mathbb{C})$ is isomorphic to the regular representation and thus it contains all the irreducible representations of $W$. For every irreducible representation $\lambda$ of $W$, consider its character $\chi^{\lambda}$. The multiplicity of $\lambda$ in the regular representation equals its degree, which we denote by $f^{\lambda}=\chi^{\lambda}(e)$. The multiplicity of the representation $\lambda$ in the representations $H^{i}(G / T ; \mathbb{C})$ for $i \geq 0$ can be described by the fake degree polynomial $f^{\lambda}(t)$ defined by

$$
f^{\lambda}(t):=\sum_{i \geq 0} t^{i}\left\langle\chi^{\lambda}, H^{i}(G / T ; \mathbb{C})\right\rangle .
$$


In other words, the coefficient of $t^{i}$ in $f^{\lambda}(t)$ is exactly the multiplicity of $\lambda$ in $H^{i}(G / T ; \mathbb{C})$. The Poincaré polynomial of the flag manifold $G / T$ is then given by

$$
P_{G / T}(t)=\sum_{\lambda} f^{\lambda} f^{\lambda}(t)=\sum_{\lambda} f^{\lambda}(1) f^{\lambda}(t),
$$

where $\lambda$ runs through all complex irreducible representations of $W$. On the other hand, the Poincaré polynomial of $E_{\mathrm{com}} G_{1}$ is given by

$$
P_{E_{\mathrm{com}} G_{\mathbf{1}}}(t)=\sum_{\lambda} f^{\bar{\lambda}}(t) f^{\lambda}(t)
$$

where $\lambda$ runs through all complex irreducible representations of $W$, and $\bar{\lambda}$ is the complex conjugate of $\lambda$. To see this, note that by Corollary 7.4 and the universal coefficient theorem we have $H^{*}\left(E_{\text {com }} G_{\mathbf{1}} ; \mathbb{C}\right) \cong\left(H^{*}(G / T ; \mathbb{C}) \otimes H^{*}(G / T ; \mathbb{C})\right)^{W}$. On the other hand, for each $0 \leq k \leq n$ we have an isomorphism

$$
\begin{aligned}
\left(H^{k}(G / T ; \mathbb{C}) \otimes H^{n-k}(G / T ;\right. & \mathbb{C}))^{W} \\
& \cong \operatorname{Hom}_{W}\left(\operatorname{Hom}\left(H^{k}(G / T ; \mathbb{C}), \mathbb{C}\right), H^{n-k}(G / T ; \mathbb{C})\right) .
\end{aligned}
$$

This together with Schur's lemma shows that $H^{n}\left(E_{\text {com }} G_{\mathbf{1}} ; \mathbb{C}\right)$ is a vector space over $\mathbb{C}$ of dimension

$$
\sum_{0 \leq k \leq n} \sum_{\lambda}\left\langle\chi^{\bar{\lambda}}, H^{k}(G / T ; \mathbb{C})\right\rangle\left\langle\chi^{\lambda}, H^{n-k}(G / T ; \mathbb{C})\right\rangle
$$

and thus $P_{E_{\mathrm{com}} G_{\mathbf{1}}}(t)=\sum_{\lambda} f^{\bar{\lambda}}(t) f^{\lambda}(t)$.

\section{The cases $\mathrm{SU}(n), U(n)$ and $\operatorname{Sp}(n)$}

In this section we study in detail the cohomology with rational coefficients of the space $B_{\text {com }} G$ when $G$ is one of the classical groups $\operatorname{SU}(n), U(n)$ and $\operatorname{Sp}(n)$ and also for their corresponding complexifications $\mathrm{SL}_{n}(\mathbb{C}), \mathrm{GL}_{n}(\mathbb{C})$ and $\mathrm{Sp}_{n}(\mathbb{C})$. In particular we provide explicit free bases of $H^{*}\left(B_{\mathrm{com}} G ; \mathbb{Q}\right)$ as a module over $H^{*}(B G ; \mathbb{Q})$. As in the previous section, we take the rational numbers as the ground field unless otherwise specified.

To start, recall that by [4, Proposition 2.5] we have that $\operatorname{Hom}\left(\mathbb{Z}^{n}, G\right)$ is path-connected for all $n \geq 0$ when $G$ is one of the groups $\operatorname{SU}(n), U(n)$ and $\operatorname{Sp}(n)$. Thus for such groups $G$ and their corresponding complexifications we have $B_{\mathrm{com}} G=B_{\mathrm{com}} G_{\mathbf{1}}$ and $E_{\mathrm{com}} G=E_{\mathrm{com}} G_{\mathbf{1}}$. 


\subsection{Case $G=U(n)$}

Suppose $G=U(n)$. In this case we can choose a maximal torus $T \subset U(n)$ to be the set of diagonal matrices with entries in $\mathbb{S}^{1}$. We have $H^{*}(B T) \cong \mathbb{Q}[\boldsymbol{x}]$, where $\boldsymbol{x}:=\left\{x_{1}, \ldots, x_{n}\right\}$ and $\operatorname{deg}\left(x_{i}\right)=2$ for $1 \leq i \leq n$. The Weyl group is the symmetric group $W=\Sigma_{n}$ acting by permutation on the variables $x_{1}, \ldots, x_{n}$. Therefore by Proposition 7.1 we have an isomorphism

$$
\alpha_{n}:=\alpha_{U(n)}: H^{*}\left(B_{\mathrm{com}} U(n)\right) \stackrel{\cong}{\rightrightarrows}(\mathbb{Q}[\boldsymbol{x}] \otimes \mathbb{Q}[\boldsymbol{y}])^{\Sigma_{n}} / J_{U(n)},
$$

where $\Sigma_{n}$ acts diagonally by permuting the variables $\boldsymbol{x}=\left\{x_{1}, \ldots, x_{n}\right\}$ and $\boldsymbol{y}=$ $\left\{y_{1}, \ldots, y_{n}\right\}$. The algebra $M^{\Sigma_{n}}:=(\mathbb{Q}[\boldsymbol{x}] \otimes \mathbb{Q}[\boldsymbol{y}])^{\Sigma_{n}}$ is known as the algebra of multisymmetric polynomials. In this case $J_{n}:=J_{U(n)}$ is the ideal in $M^{\Sigma_{n}}$ generated by the elementary symmetric polynomials

$$
e_{k}\left(x_{1}, \ldots, x_{n}\right)=\sum_{\substack{1 \leq i_{1}<i_{2} \\<\cdots<i_{k} \leq n}} x_{i_{1}} x_{i_{2}} \cdots x_{i_{k}}
$$

for $1 \leq k \leq n$. Since we are working with rational coefficients, the ideal $J_{n}$ is also the ideal generated by the power sums $p_{n}(a, 0):=x_{1}^{a}+\cdots+x_{n}^{a}$ for $1 \leq a \leq n$. These classical power sums have analogues in the ring of multisymmetric polynomials. For every pair of integers $a, b \geq 0$ define the power sum $p_{n}(a, b):=x_{1}^{a} y_{1}^{b}+\cdots+$ $x_{n}^{a} y_{n}^{b}$. Clearly $p_{n}(a, b) \in M^{\Sigma_{n}}$ for all $a, b \geq 0$. Moreover, it is well known that the polynomials $p_{n}(a, b)$ for $0<a+b \leq n$ generate the $\mathbb{Q}$-algebra $M^{\Sigma_{n}}$ although these polynomials are not algebraically independent. (See for example [27] for a modern account on multisymmetric polynomials). We know by Theorem 7.2 that $M^{\Sigma_{n}} / J_{n}$ is a free module over $H^{*}(B U(n)) \cong \mathbb{Q}[\boldsymbol{y}]^{\Sigma_{n}}$. An explicit free basis for $M^{\Sigma_{n}} / J_{n}$ as a module over $\mathbb{Q}[\boldsymbol{y}]^{\Sigma_{n}}$ can be constructed using the work in [7]. For this consider the averaging operator

$$
\begin{aligned}
\rho: \mathbb{Q}[\boldsymbol{x}] \otimes \mathbb{Q}[\boldsymbol{y}] & \rightarrow(\mathbb{Q}[\boldsymbol{x}] \otimes \mathbb{Q}[\boldsymbol{y}])^{\Sigma_{n}}=M^{\Sigma_{n}}, \\
f(\boldsymbol{x}, \boldsymbol{y}) & \mapsto \frac{1}{n !} \sum_{w \in \Sigma_{n}} f(w \boldsymbol{x}, w \boldsymbol{y}) .
\end{aligned}
$$

For every $w \in \Sigma_{n}$ the diagonal descent monomial is defined to be

$$
e_{w}:=\prod_{\substack{w^{-1}(i) \\>w^{-1}(i+1)}}\left(x_{1} \cdots x_{i}\right) \otimes \prod_{\substack{w(j) \\>w(j+1)}}\left(y_{w(1)} \cdots y_{w(j)}\right) .
$$

By [7, Theorem 1.3] the collection $\left\{\rho\left(e_{w}\right)\right\}_{w \in \Sigma_{n}}$ forms a free basis of $M^{\Sigma_{n}}$ as a module over $\mathbb{Q}[\boldsymbol{x}]^{\Sigma_{n}} \otimes \mathbb{Q}[\boldsymbol{y}]^{\Sigma_{n}}$. 
Example 8.1 Suppose that $n=3$. In this case we obtain the following basis of $M^{\Sigma_{3}}$ as a module over $\mathbb{Q}[\boldsymbol{x}]^{\Sigma_{3}} \otimes \mathbb{Q}[\boldsymbol{y}]^{\Sigma_{3}}$ :

$$
\begin{array}{lll}
e_{1}=1, & e_{2}=\rho\left(x_{1} y_{2}\right), & e_{3}=\rho\left(x_{1} y_{2} y_{3}\right), \\
e_{4}=\rho\left(x_{1} x_{2} y_{3}\right), & e_{5}=\rho\left(x_{1} x_{2} y_{1} y_{3}\right), & e_{6}=\rho\left(x_{1}^{2} x_{2} y_{3}^{2} y_{2}\right) .
\end{array}
$$

Let $f_{w}$ be the image of $\rho\left(e_{w}\right)$ in $M^{\Sigma_{n}} / J_{n}$. Then it follows that $\left\{f_{w}\right\}_{w \in \Sigma_{n}}$ forms a free basis of $H^{*}\left(B_{\text {com }} U(n)\right) \cong M^{\Sigma_{n}} / J_{n}$ as a module over $H^{*}(B U(n)) \cong \mathbb{Q}[\boldsymbol{y}]^{\Sigma_{n}}$. For each $w \in \Sigma_{n}$ define the descent of $w$ to be the set

$$
\operatorname{Des}(w):=\{1 \leq i \leq n-1 \mid w(i)>w(i+1)\} .
$$

The major index of $w$, denoted by maj $(w)$, is defined to be

$$
\operatorname{maj}(w):=\sum_{i \in \operatorname{Des}(w)} i=\sum_{w(i)>w(i+1)} i .
$$

For every $w \in \Sigma_{n}$ we have $\operatorname{deg} f_{w}=2\left(\operatorname{maj}(w)+\operatorname{maj}\left(w^{-1}\right)\right)$. As a corollary we obtain the following.

Corollary 8.2 Suppose that $n \geq 1$. Then

$$
\begin{aligned}
& P_{E_{\mathrm{com}} \mathrm{GL}_{n}(\mathbb{C})}=P_{E_{\mathrm{com}} U(n)}(t)=\sum_{w \in \Sigma_{n}} t^{2\left(\operatorname{maj}(w)+\operatorname{maj}\left(w^{-1}\right)\right)}, \\
& P_{B_{\mathrm{com}} \mathrm{GL}_{n}(\mathbb{C})}=P_{B_{\mathrm{com}} U(n)}(t)=\frac{\sum_{w \in \Sigma_{n}} t^{2\left(\operatorname{maj}(w)+\operatorname{maj}\left(w^{-1}\right)\right)}}{\prod_{1 \leq i \leq n}\left(1-t^{2 i}\right)} .
\end{aligned}
$$

Consider now the standard inclusion $i_{n}: U(n) \rightarrow U(n+1)$. Let $U:=\operatorname{colim}_{n \rightarrow \infty} U(n)$. Then $B_{\text {com }} U=\operatorname{colim}_{n \rightarrow \infty} B_{\text {com }} U(n)$ and $H^{*}\left(B_{\text {com }} U(n)\right) \cong \lim _{\longleftarrow} H^{*}\left(B_{\text {com }} U(n)\right)$. The isomorphisms $\alpha_{n}$ and the maps $i_{n}$ are compatible in the sense that

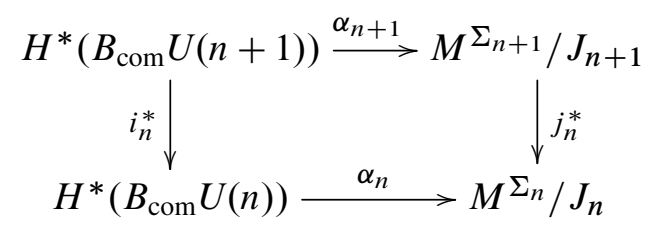

is a commutative diagram, where $j_{n}^{*}: M^{\Sigma_{n+1}} \rightarrow M^{\Sigma_{n}}$ is the map obtained by sending $x_{i} \mapsto x_{i}, y_{i} \mapsto y_{i}$ for $1 \leq i \leq n$ and $x_{n+1}, y_{n+1} \mapsto 0$. Define

$$
M^{\Sigma_{\infty}}:=\lim _{\longleftarrow} M^{\Sigma_{n}} \text { and } J_{\infty}:=\lim _{\longleftarrow} J_{n} .
$$


Then we obtain an isomorphism of graded $\mathbb{Q}$-algebras

$$
H^{*}\left(B_{\mathrm{com}} U\right) \cong \lim ^{\Sigma_{n}} J_{n} \cong M^{\Sigma_{\infty}} / J_{\infty} .
$$

The last isomorphism follows from the fact that $j_{n}^{*}: J_{n+1} \rightarrow J_{n}$ is surjective for every $n \geq 1$ and thus $\lim ^{1} J_{n}$ vanishes. We show next that this algebra is a polynomial algebra. For every pair of integers $a, b \geq 0$ we have $j_{n}^{*}\left(p_{n+1}(a, b)\right)=p_{n}(a, b)$ and thus these polynomials define an element in $M^{\Sigma_{\infty}}$, which we denote by $p(a, b)$. By [27, Theorem 2] the algebra $M^{\Sigma_{\infty}}$ is a polynomial algebra over $\mathbb{Q}$ generated by the elements $p(a, b)$ for $(a, b) \neq 0$. On the other hand, since $J_{n}$ is the ideal in $M^{\Sigma_{n}}$ generated by the power sums $p_{n}(1,0), \ldots, p_{n}(n, 0)$, it follows that $J_{\infty}$ is the ideal generated by $p(a, 0)$ for $a \geq 1$. For each pair of integers $a, b \geq 0$ not both zero let $z_{a, b}$ be a $2(a+b)$-dimensional variable such that the collection $\left\{z_{a, b}\right\}_{a, b}$ is a collection of commuting independent variables. Define $\mathfrak{M}_{U}=\left\{(a, b) \in \mathbb{N}^{2} \mid b>0\right\}$; we conclude that the assignment

$$
\begin{aligned}
\mathbb{Q}\left[z_{a, b} \mid(a, b) \in \mathfrak{M}_{U}\right] & \rightarrow M^{\Sigma_{\infty}} / J_{n} \cong H^{*}\left(B_{\mathrm{com}} U\right), \\
z_{a, b} & \mapsto p(a, b)
\end{aligned}
$$

is an isomorphism of algebras over $\mathbb{Q}$. Also define $\mathrm{GL}_{\infty}(\mathbb{C}):=\operatorname{colim}_{n \rightarrow \infty} \mathrm{GL}_{n}(\mathbb{C})$. Since $B_{\text {com }} U(n) \simeq B_{\text {com }} \mathrm{GL}_{n}(\mathbb{C})$ for every $n \geq 0$, it follows that

$$
B_{\mathrm{com}} U \simeq B_{\mathrm{com}} \mathrm{GL}_{\infty}(\mathbb{C}) \text {. }
$$

This proves the following corollary.

Corollary 8.3 Let $\mathfrak{M}_{U}=\left\{(a, b) \in \mathbb{N}^{2} \mid b>0\right\}$. Then we have isomorphisms of $\mathbb{Q}$-algebras

$$
H^{*}\left(B_{\mathrm{com}} \mathrm{GL}_{\infty}(\mathbb{C})\right) \cong H^{*}\left(B_{\mathrm{com}} U\right) \cong \mathbb{Q}\left[z_{a, b} \mid(a, b) \in \mathfrak{M}_{U}\right]
$$

\subsection{Case $G=\mathrm{SU}(n)$}

The case of the special unitary groups $G=\mathrm{SU}(n)$ can be handled in a similar way. In this case we can choose $T \subset \mathrm{SU}(n)$ to be the set of diagonal matrices with entries in $\mathbb{S}^{1}$ and determinant one. The Weyl group is the symmetric group $W=\Sigma_{n}$ acting by permutation on the diagonal entries and $H^{*}(B T) \cong \mathbb{Q}[\boldsymbol{x}] /\left(p_{n}(1,0)\right)$, where as before we use the notation $\boldsymbol{x}=\left\{x_{1}, \ldots, x_{n}\right\}$. Using an argument similar to that in Proposition 7.1, we conclude that

$$
H^{*}\left(B_{\mathrm{com}} \mathrm{SU}(n)\right) \cong(\mathbb{Q}[\boldsymbol{x}] \otimes \mathbb{Q}[\boldsymbol{y}])^{\Sigma_{n}} / K_{n}=M^{\Sigma_{n}} / K_{n},
$$

where $K_{n}$ is the ideal in $M^{\Sigma_{n}}$ generated by the multisymmetric polynomials $p_{n}(a, 0)$ for $1 \leq a \leq n$ and $p_{n}(0,1)$. As it was pointed out before the collection $\left\{\rho\left(e_{w}\right)\right\}_{w \in \Sigma_{n}}$ 
forms a free basis for $M^{\Sigma_{n}}$ as a module over $\mathbb{Q}[x]^{\Sigma_{n}} \otimes \mathbb{Q}[\boldsymbol{y}]^{\Sigma_{n}}$. Let $g_{w}$ denote the image of $\rho\left(e_{w}\right)$ in $M^{\Sigma_{n}} / K_{n}$. Then it follows that $\left\{g_{w}\right\}_{w \in \Sigma_{n}}$ forms a free basis for $H^{*}\left(B_{\text {com }} \mathrm{SU}(n)\right) \cong M^{\Sigma_{n}} / K_{n}$ as a module over

$$
H^{*}(B \mathrm{SU}(n))=\mathbb{Q}\left[p_{n}(0,2), \ldots, p_{n}(0, n)\right] .
$$

As a corollary we get the following.

Corollary 8.4 Suppose that $n \geq 1$. Then

$$
\begin{aligned}
& P_{E_{\mathrm{com}} \mathrm{SL}_{n}(\mathbb{C})}(t)=P_{E_{\mathrm{com}} \mathrm{SU}(n)}(t)=\sum_{w \in \Sigma_{n}} t^{2\left(\operatorname{maj}(w)+\operatorname{maj}\left(w^{-1}\right)\right)}, \\
& P_{B_{\mathrm{com}} \mathrm{SL}_{n}(\mathbb{C})}(t)=P_{B_{\mathrm{com}} \mathrm{SU}(n)}(t)=\frac{\sum_{w \in \Sigma_{n}} t^{2\left(\operatorname{maj}(w)+\operatorname{maj}\left(w^{-1}\right)\right)}}{\prod_{2 \leq i \leq n}\left(1-t^{2 i}\right)},
\end{aligned}
$$

where maj $(w)$ is the major index of $w$ as defined above.

As in the case of the unitary groups we have a stabilization process given by the standard inclusions $i_{n}: \mathrm{SU}(n) \rightarrow \mathrm{SU}(n+1)$. Let $\mathrm{SU}:=\operatorname{colim}_{n \rightarrow \infty} \mathrm{SU}(n)$. It follows that

$$
H^{*}\left(B_{\mathrm{com}} \mathrm{SU}\right)=\lim _{\longleftarrow} H^{*}\left(B_{\mathrm{com}} \mathrm{SU}(n)\right)=\lim _{\longleftarrow} M^{\Sigma_{n}} / K_{n} .
$$

Let $K_{\infty} \subset M^{\Sigma_{\infty}}$ denote the ideal corresponding to the ideals $K_{n} \subset M^{\Sigma_{n}}$ for $n \geq 0$. For SU we have $H^{*}\left(B_{\text {com }} \mathrm{SU}\right) \cong M^{\Sigma_{\infty}} / K_{\infty}$. Note that $K_{\infty}$ is precisely the ideal in $M^{\Sigma_{\infty}}$ generated by $p(a, 0)$ for $a \geq 1$ and $p(0,1)$. Similarly define $\operatorname{SL}_{\infty}(\mathbb{C}):=$ colim $_{n \rightarrow \infty} \operatorname{SL}_{n}(\mathbb{C})$. As a corollary we get the following.

Corollary 8.5 Let $\mathfrak{M}_{\mathrm{SU}}=\left\{(a, b) \in \mathbb{N}^{2} \mid(a, b) \neq(0,1), b>0\right\}$. Then we have isomorphisms of $\mathbb{Q}$-algebras

$$
H^{*}\left(B_{\mathrm{com}} \mathrm{SL}_{\infty}(\mathbb{C})\right) \cong H^{*}\left(B_{\mathrm{com}} \mathrm{SU}\right) \cong \mathbb{Q}\left[z_{a, b} \mid(a, b) \in \mathfrak{M}_{\mathrm{SU}}\right]
$$

\subsection{Case $G=\operatorname{Sp}(n)$}

Finally, suppose that $G=\operatorname{Sp}(n)$. In this case $H^{*}(B T) \cong \mathbb{Q}[\boldsymbol{x}]$, where $\boldsymbol{x}=\left\{x_{1}, \ldots, x_{n}\right\}$ and $\operatorname{deg}\left(x_{i}\right)=2$. The Weyl group is a semi-direct product $W=\Sigma_{n} \ltimes(\mathbb{Z} / 2)^{n}$. This group can be identified with the group of signed permutations. More precisely, let

$$
\mathbb{I}_{n}:=\{-n,-n+1, \ldots,-1,1, \ldots, n-1, n\} .
$$

Let $B_{n}$ denote the group of bijections $\sigma: \mathbb{I}_{n} \rightarrow \mathbb{I}_{n}$ such that $\sigma(-k)=-\sigma(k)$ for all $k \in$ $\mathbb{I}_{n}$, with the composition of functions as the group operation. Under this identification the group $W \cong B_{n}$ acts on $\mathbb{Q}[\boldsymbol{x}]$ by signed permutations. In this case $H^{*}(B G)$ is a 
polynomial algebra generated by $e_{1}\left(x_{1}^{2}, \ldots, x_{n}^{2}\right), \ldots, e_{n}\left(x_{1}^{2}, \ldots, x_{n}^{2}\right)$, or equivalently, by the power sums $p_{n}(2,0), \ldots, p_{n}(2 n, 0)$. Also $M^{B_{n}}:=(\mathbb{Q}[\boldsymbol{x}] \otimes \mathbb{Q}[\boldsymbol{y}])^{\boldsymbol{B}_{n}}$ is the ring of diagonally signed-symmetric or signed-invariant multisymmetric polynomials. Note in particular that $M^{B_{n}}$ is a subalgebra of the algebra of multisymmetric polynomials $M^{\Sigma_{n}}$. By Proposition 7.1 we have an isomorphism

$$
\alpha_{\mathrm{Sp}(n)}: H^{*}\left(B_{\mathrm{com}} \operatorname{Sp}(n)\right) \stackrel{\cong}{\rightrightarrows} M^{B_{n}} / L_{n},
$$

where $L_{n}=J_{\mathrm{Sp}(n)}$ is the ideal in $M^{B_{n}}$ generated by the power sums $p_{n}(2,0), \ldots$, $p_{n}(2 n, 0)$. An explicit basis for $M^{B_{n}} / L_{n}$ as a module over $\mathbb{Q}[\boldsymbol{y}]^{B_{n}}$ can be found using the work in [12]. As before, given $w \in B_{n}$, define its descent to be the set

$$
\operatorname{Des}(w):=\{1 \leq i \leq n-1 \mid w(i)>w(i+1)\} .
$$

For $1 \leq i \leq n$ let

$$
\begin{aligned}
& d_{i}(w):=|\{j \in \operatorname{Des}(w) \mid j \geq i\}|, \\
& f_{i}(w):=2 d_{i}(w)+\varepsilon_{i}(w),
\end{aligned} \quad \varepsilon_{i}(w):= \begin{cases}0 & \text { if } w(i)>0, \\
1 & \text { if } w(i)<0 .\end{cases}
$$

The diagonal signed descent monomial associated to $w$ is defined to be

$$
c_{w}:=\left(\prod_{i=1}^{n} x_{i}^{f_{i}\left(w^{-1}\right)}\right)\left(\prod_{i=1}^{n} y_{|w(i)|}^{f_{i}(w)}\right)=\prod_{i=1}^{n} x_{i}^{f_{i}\left(w^{-1}\right)} y_{i}^{f_{\left|w^{-1}(i)\right|}(w)} .
$$

By [12, Theorem 1.1] the collection $\left\{\rho\left(c_{w}\right)\right\}_{w \in \Sigma_{n}}$ forms a free basis of $M^{B_{n}}$ as a module over $\mathbb{Q}[\boldsymbol{x}]^{B_{n}} \otimes \mathbb{Q}[\boldsymbol{y}]^{B_{n}}$.

Example 8.6 Suppose that $n=2$. In this case we obtain the following basis of $M^{B_{2}}$ as a module over $\mathbb{Q}[\boldsymbol{x}]^{B_{2}} \otimes \mathbb{Q}[\boldsymbol{y}]^{B_{2}}$ :

$$
\begin{array}{llll}
c_{1}=1, & c_{2}=\rho\left(x_{1} y_{1}\right), & c_{3}=\rho\left(x_{1}^{2} y_{2}^{2}\right), & c_{4}=\rho\left(x_{1} y_{1} y_{2}^{2}\right), \\
c_{5}=\rho\left(x_{1}^{2} x_{2} y_{2}\right), & c_{6}=\rho\left(x_{1} x_{2} y_{1} y_{2}\right), & c_{7}=\rho\left(x_{1}^{2} x_{2} y_{1}^{2} y_{2}\right), & c_{8}=\rho\left(x_{1}^{3} x_{2} y_{1}^{3} y_{2}\right) .
\end{array}
$$

Let $h_{w}$ be the image of $\rho\left(c_{w}\right)$ in $M^{B_{n}} / L_{n}$. It follows that $\left\{h_{w}\right\}_{w \in B_{n}}$ forms a free basis of $H^{*}\left(B_{\text {com }} \operatorname{Sp}(n)\right) \cong M^{B_{n}} / L_{n}$ as a module over $H^{*}(B \operatorname{Sp}(n)) \cong \mathbb{Q}[y]^{B_{n}}$. The flag major index of a signed permutation $w$ was defined in [6] to be

$$
\operatorname{fmaj}(w)=\sum_{i=1}^{n} f_{i}(w)=2 \operatorname{maj}(w)+\operatorname{neg}(w),
$$

where $\operatorname{maj}(w):=\sum_{i \in \operatorname{Des}(w)} i=\sum_{w(i)>w(i+1)} i$ and $\operatorname{neg}(w):=|\{1 \leq i \leq n \mid w(i)<0\}|$. Note that for every $w \in B_{n}$ we have $\operatorname{deg} h_{w}=2\left(\operatorname{fmaj}\left(w^{-1}\right)+\operatorname{fmaj}(w)\right)$. As a corollary we get the following. 
Corollary 8.7 Suppose that $n \geq 1$. Then

$$
\begin{aligned}
& P_{E_{\mathrm{com} S \mathrm{Sp}_{n}}(\mathbb{C})}(t)=P_{E_{\mathrm{com}} \mathrm{Sp}(n)}(t)=\sum_{w \in B_{n}} t^{2\left(\mathrm{fmaj}\left(w^{-1}\right)+\mathrm{fmaj}(w)\right)}, \\
& P_{B_{\mathrm{com} S \mathrm{Sp}_{n}}(\mathbb{C})}(t)=P_{B_{\mathrm{com} S}(n)}(t)=\frac{\sum_{w \in B_{n}} t^{2\left(\mathrm{fmaj}\left(w^{-1}\right)+\mathrm{fmaj}(w)\right)}}{\prod_{1 \leq i \leq n}\left(1-t^{4 i}\right)} .
\end{aligned}
$$

As in the case of the unitary groups we have a stabilization process given by the standard inclusions $i_{n}: \operatorname{Sp}(n) \rightarrow \operatorname{Sp}(n+1)$. Let $\mathrm{Sp}:=\operatorname{colim}_{n \rightarrow \infty} \operatorname{Sp}(n)$. Recall that we have an isomorphism $H^{*}\left(B_{\text {com }} \operatorname{Sp}(n)\right) \cong M^{B_{n}} / L_{n}$, where $L_{n}=J_{\mathrm{Sp}(n)}$ is the ideal in $M^{B_{n}}$ generated by the power sums $p_{n}(2,0), \ldots, p_{n}(2 n, 0)$. Thus for $\mathrm{Sp}$ we have

$$
H^{*}\left(B_{\mathrm{com}} \mathrm{Sp}\right) \cong \lim _{\longleftarrow} H^{*}\left(B_{\mathrm{com}} \mathrm{Sp}(n)\right) \cong \lim M^{B_{n}} / L_{n} .
$$

Define

$$
M^{B_{\infty}}=\lim _{\longleftarrow} M^{B_{n}} \quad \text { and } \quad L_{\infty}:=\lim _{\longleftarrow} L_{n} .
$$

Thus for the group Sp we have an isomorphism of algebras over $\mathbb{Q}$

$$
H^{*}\left(B_{\mathrm{com}} \mathrm{Sp}\right) \cong M^{B} / L_{\infty}
$$

Next we show that $M^{B} \infty$ is a polynomial algebra. To see this we first show that the power sums $p_{n}(a, b)=x_{1}^{a} y_{1}^{b}+\cdots+x_{n}^{a} y_{n}^{b}$, where $a, b$ runs though all nonnegative integers such that $0<a+b$ and $a+b$ is even, generate $M^{B_{n}}$ as a $\mathbb{Q}$-algebra. For this, consider the averaging operator $\rho: \mathbb{Q}[\boldsymbol{x}, \boldsymbol{y}] \rightarrow \mathbb{Q}[\boldsymbol{x}, \boldsymbol{y}]^{B_{n}}$ corresponding to the group $B_{n}$. Note that as a $\mathbb{Q}$-vector space $M^{B_{n}}$ is generated by the elements of the form $\rho(m(\boldsymbol{x}, \boldsymbol{y}))$, where $m(\boldsymbol{x}, \boldsymbol{y})=x_{1}^{i_{1}} y_{1}^{j_{1}} \cdots x_{n}^{i_{n}} y_{n}^{j_{n}}$ is a monomial. By [12, Lemma 3.2] if $i_{k}+j_{k}$ is odd for some $1 \leq k \leq n$ then $\rho(m(\boldsymbol{x}, \boldsymbol{y}))=0$. It follows that as a $\mathbb{Q}$-vector space $M^{B_{n}}$ is generated by the elements of the form $\rho(m(\boldsymbol{x}, \boldsymbol{y}))$, where $m(\boldsymbol{x}, \boldsymbol{y})=x_{1}^{i_{1}} y_{1}^{j_{1}} \cdots x_{n}^{i_{n}} y_{n}^{j_{n}}$ and $i_{k}+j_{k}$ is even for $1 \leq k \leq n$. Suppose that $m(\boldsymbol{x}, \boldsymbol{y})$ is such a monomial. Define the length of $m(\boldsymbol{x}, \boldsymbol{y}), \ell(m(\boldsymbol{x}, \boldsymbol{y}))$, to be the number of tuples $\left(i_{k}, j_{k}\right)$ that are nonzero for $1 \leq k \leq n$. We can show that $\rho(m(\boldsymbol{x}, \boldsymbol{y}))$ is a polynomial on the different $p_{n}(a, b)$ in an inductive way on the length of the monomial $m(\boldsymbol{x}, \boldsymbol{y})$. If $\ell(m(\boldsymbol{x}, \boldsymbol{y}))=1$ we have

$$
\rho(m(\boldsymbol{x}, \boldsymbol{y}))=\frac{1}{n}\left(x_{1}^{i} y_{1}^{j}+\cdots+x_{n}^{i} y_{n}^{j}\right)=\frac{p_{n}(i, j)}{n}
$$

and there is nothing to prove. Given any monomial $m(\boldsymbol{x}, \boldsymbol{y})=x_{1}^{i_{1}} y_{1}^{j_{1}} \cdots x_{n}^{i_{n}} y_{n}^{j_{n}}$ with length $\ell(m(\boldsymbol{x}, \boldsymbol{y}))=r$, let $\left(i_{k_{1}}, j_{k_{1}}\right), \ldots,\left(i_{k_{r}}, j_{k_{r}}\right)$ be the corresponding different 
tuples that are nonzero. Note that

$$
\begin{aligned}
p_{n}\left(i_{k_{1}}, j_{k_{1}}\right) \cdots p_{n}\left(i_{k_{r}}, j_{k_{r}}\right) & =\left(\sum_{i=1}^{n} x_{i}^{i_{k_{1}}} y_{i}^{j_{k_{1}}}\right) \cdots\left(\sum_{i=1}^{n} x_{i}^{i_{k r}} y_{i}^{j_{k r}}\right) \\
& =c \rho(m(\boldsymbol{x}, \boldsymbol{y}))+q(\boldsymbol{x}, \boldsymbol{y}),
\end{aligned}
$$

where $c$ is a nonzero constant and $q(\boldsymbol{x}, \boldsymbol{y})$ is a sum of certain monomials $n(\boldsymbol{x}, \boldsymbol{y})$ with $\ell(n(\boldsymbol{x}, \boldsymbol{y}))<r$. This proves that the elements $p_{n}(a, b)$, where $a+b>0$ is even, generate $M^{B_{n}}$. In fact it can be seen that the collection $\left\{p_{n}(a, b)\right\}$, where $a, b$ run through all the nonnegative integers such that $0<a+b \leq 2 n$ and $a+b$ is even, generate $M^{B_{n}}$, but we do not need that fact. Recall that for every $n \geq 1$ we have a map $j_{n}^{*}: M^{B_{n+1}} \rightarrow M^{B_{n}}$ obtained by sending $x_{i} \mapsto x_{i}, y_{i} \mapsto y_{i}$ for $1 \leq i \leq n$ and $x_{n+1}, y_{n+1} \mapsto 0$ and $M^{B_{\infty}}:=\lim M^{B_{n}}$. Suppose that $a, b$ are nonnegative integers. Note that $j_{n}^{*}\left(p_{n+1}(a, b)\right)=p_{n}(a, b)$ and thus the different polynomials $p_{n}(a, b)$ induce an element $p(a, b)$ in $M^{B_{\infty}}$. Note that each signed-multisymmetric polynomial is in particular a multisymmetric polynomial; that is, we can see $M^{B_{\infty}}$ as a subset of $M^{\Sigma_{\infty}}$. Also, we know that $M^{\Sigma_{\infty}}$ is a polynomial algebra on the different elements $p(a, b)$ where $a+b>0$ by [27, Theorem 2]. This implies in particular that the collection $\{p(a, b)\}_{a+b>0 \text {,even }}$ is algebraically independent in $M^{\Sigma_{\infty}}$ and in particular, it is also algebraically independent in $M^{B}$. As a corollary we obtain:

Corollary 8.8 The $\mathbb{Q}$-algebra $M^{B_{\infty}}$ is a polynomial algebra on the generators $p(a, b)$, where $a, b$ run through all nonnegative integers such that $0<a+b$ is even.

Using the previous fact we can obtain a description of $H^{*}\left(B_{\mathrm{com}} \mathrm{Sp}\right)$ as an algebra. Indeed, recall that

$$
H^{*}\left(B_{\mathrm{com}} \mathrm{Sp}\right) \cong M^{B} / L_{\infty},
$$

where $L_{\infty}$ is the ideal generated by the power sums $p(2 n, 0)$ for all $n \geq 1$. Similarly define $\operatorname{Sp}_{\infty}(\mathbb{C})=\operatorname{colim}_{n \rightarrow \infty} \operatorname{Sp}_{n}(\mathbb{C})$. As a corollary we obtain the following.

Corollary 8.9 Define $\mathfrak{M}_{\mathrm{Sp}}=\left\{(a, b) \in \mathbb{N}^{2} \mid b>0, a+b\right.$ even $\}$. Then we have isomorphisms of $\mathbb{Q}$-algebras

$$
H^{*}\left(B_{\mathrm{com}} \operatorname{Sp}_{\infty}(\mathbb{C})\right) \cong H^{*}\left(B_{\mathrm{com}} \mathrm{Sp}\right) \cong \mathbb{Q}\left[z_{a, b} \mid(a, b) \in \mathfrak{M}_{\mathrm{Sp}}\right]
$$

\section{Appendix}

The goal of this appendix is to show that for any Lie group $G$ the simplicial space $\left[B_{\mathrm{com}} G\right]_{*}$ is a proper simplicial space. This fact was proved in [1, Theorem 8.3] for 
Lie groups $G$ that are closed subgroups of $\mathrm{GL}_{n}(\mathbb{C})$ for some $n \geq 0$ and extended in the equivariant setting in [2] for compact Lie groups. Here we show that the arguments in [1] can be used to proved this result for any Lie group $G$.

We start by recalling some basic definitions. Recall that a pair of topological spaces $(X, A)$ is said to be an NDR pair if there exist continuous functions $h: X \times[0,1] \rightarrow X$ and $u: X \rightarrow[0,1]$ such that the following conditions are satisfied:

(1) $A=u^{-1}(0)$.

(2) $h(x, 0)=x$ for all $x \in X$.

(3) $h(a, t)=a$ for all $a \in A$ and all $t \in[0,1]$.

(4) $h(x, 1) \in A$ for all $x \in u^{-1}([0,1))$.

In this case we say that $(h, u)$ is a representation of $(X, A)$ as an NDR pair. If in addition we have $u(h(x, t))<1$ for all $t \in[0,1]$ whenever $u(x)<1$, then $(X, A)$ is called a strong NDR pair. A simplicial space $X_{*}$ is said to be proper if each pair $\left(X_{n+1}, s X_{n}\right)$ is a strong NDR pair, where $s X_{n}$ is the image of the different degeneracy maps in $X_{n+1}$.

Proposition A.1 If $G$ is a Lie group then $\left[B_{\mathrm{com}} G\right]_{*}$ is a proper simplicial space.

Proof Suppose that $G$ is a Lie group and let $\mathfrak{g}$ denote its Lie algebra endowed with a norm $\|\cdot\|_{\mathfrak{g}}$. Recall that the exponential map exp: $\mathfrak{g} \rightarrow G$ is a local homeomorphism. Let $U \subset \mathfrak{g}$ be any $A d$-invariant open neighborhood of $0 \in \mathfrak{g}$ on which the exponential map is injective. Fix some $\epsilon>0$ such $\bar{B}_{\epsilon}(0) \subset U$. Then in particular $\exp _{\mid}: \bar{B}_{\epsilon}(0) \rightarrow G$ is a homeomorphism onto its image. Define a function $u: G \rightarrow[0,1]$ by

$$
u(g)= \begin{cases}2|y|_{\mathfrak{g}} / \epsilon & \text { if } g=\exp (y) \text { for } g \in \exp \left(\bar{B}_{\epsilon / 2}(0)\right), \\ 1 & \text { if } g \in G-\exp \left(B_{\epsilon / 2}(0)\right) .\end{cases}
$$

Also, let $s: G \rightarrow[0,1]$ be any bump function satisfying the following conditions

$$
s(g)= \begin{cases}1 & \text { if } g=\exp (y) \text { for } g \in \exp \left(\bar{B}_{\epsilon / 2}(0)\right), \\ 0 & \text { if } g \in G-\exp \left(B_{\epsilon}(0)\right) .\end{cases}
$$

Finally, define a homotopy $h: G \times[0,1] \rightarrow G$ by

$$
h(g, t)= \begin{cases}\exp ((1-t) y) & \text { if } g=\exp (y) \text { for } y \in \bar{B}_{\epsilon / 2}(0), \\ \exp ((1-s(g) t) y) & \text { if } g=\exp (y) \text { for } y \in \bar{B}_{\epsilon}(0)-B_{\epsilon / 2}(0), \\ g & \text { if } g \in G-\exp \left(B_{\epsilon}(0)\right)\end{cases}
$$

The functions $h$ and $u$ are defined so that $(h, u)$ is representation of $\left(G,\left\{1_{G}\right\}\right)$ as an NDR pair. This can be seen in the same way as in [1, Proposition 8.2]. Moreover, 
we claim that the function $h$ satisfies the following property: for each $g \neq 1_{G}$ in $G$ and each $0 \leq t<1$ we have $Z_{G}(h(g, t))=Z_{G}(g)$. Indeed, assume that $g \in G$ with $g \neq 1_{G}$. Note that if $0 \leq t<1$ then $h(g, t)=g$ if $g \in G-\exp \left(B_{\epsilon}(0)\right)$ and $h(g, t)=\exp (k y)$ for some $0<k \leq 1$ if $g=\exp (y)$ with $y \in \exp \left(B_{\epsilon}(0)\right)$. In the first case we have nothing to prove. Suppose then that $g=\exp (y)$ with $y \in \exp \left(B_{\epsilon}(0)\right)$ and thus $h(g, t)=\exp (k y)$ for some $0<k \leq 1$. Since $y \in \exp \left(B_{\epsilon}(0)\right) \subset U$ and $U$ is an $A d$-invariant open set on which the exponential map is injective, then by [11, Lemma 3.2.1] we have $Z_{G}(g)=Z_{G}(\exp (y))=Z_{G}(\exp (k y))=Z_{G}(h(g, t))$ proving that $Z_{G}(g)=Z_{G}(h(g, t))$ as claimed. By [1, Theorem 7.3] we conclude that the inclusion map $I_{j}: S_{n}(j, G) \hookrightarrow S_{n}(j-1, G)$ is a cofibration for every $1 \leq j \leq n$ (in the terminology of [1, Definition 6.1], $G$ has cofibrantly commuting elements). Here $S_{n}(j, G)$ denotes the subspace of $\operatorname{Hom}\left(\mathbb{Z}^{n}, G\right)$ consisting of the commuting $n$-tuples with at least $j$ coordinates equal to $1_{G}$. This implies in particular that the inclusion map $s\left(\left[B_{\mathrm{com}} G\right]_{n-1}\right)=S_{n}(1, G) \hookrightarrow \operatorname{Hom}\left(\mathbb{Z}^{n}, G\right)=\left[B_{\mathrm{com}} G\right]_{n}$ is a cofibration. Using the explicit NDR-pair representation of $\left(\operatorname{Hom}\left(\mathbb{Z}^{n}, G\right), S_{n}(1, G)\right)$ provided by $\left[1\right.$, Theorem 7.3] it can easily be seen that $\left(\operatorname{Hom}\left(\mathbb{Z}^{n}, G\right), S_{n}(1, G)\right)$ is actually a strong $\mathrm{NDR}$-pair, proving that $\left[B_{\mathrm{com}} G\right]_{*}$ is a proper simplicial space.

Remark A.2 Using the explicit NDR representation provided by [1, Theorem 7.3] for the pair $\left(\operatorname{Hom}\left(\mathbb{Z}^{n}, G\right), S_{n}(1, G)\right)$, it can be seen that in fact $\left[B_{\mathrm{com}} G\right]_{*}$ is a strictly proper simplicial space (see [18, Definition 11.2] for the definition of a strictly proper simplicial space).

\section{References}

[1] A Adem, F R Cohen, Commuting elements and spaces of homomorphisms, Math. Ann. 338 (2007) 587-626 MR2317932

[2] A Adem, F R Cohen, J M Gómez, Stable splittings, spaces of representations and almost commuting elements in Lie groups, Math. Proc. Cambridge Philos. Soc. 149 (2010) 455-490 MR2726729

[3] A Adem, F R Cohen, E Torres-Giese, Commuting elements, simplicial spaces and filtrations of classifying spaces, Math. Proc. Cambridge Philos. Soc. 152 (2012) 91-114 MR2860418

[4] A Adem, J M Gómez, Equivariant K-theory of compact Lie group actions with maximal rank isotropy, J. Topol. 5 (2012) 431-457 MR2928083

[5] A Adem, J M Gómez, J A Lind, U Tillmann, Infinite loop spaces and nilpotent $K_{-}$ theory, in preparation

[6] R M Adin, Y Roichman, The flag major index and group actions on polynomial rings, European J. Combin. 22 (2001) 431-446 MR1829737 
[7] E E Allen, The descent monomials and a basis for the diagonally symmetric polynomials, J. Algebraic Combin. 3 (1994) 5-16 MR1256100

[8] T J Baird, Cohomology of the space of commuting $n$-tuples in a compact Lie group, Algebr. Geom. Topol. 7 (2007) 737-754 MR2308962

[9] A Borel, Sous-groupes commutatifs et torsion des groupes de Lie compacts connexes, Tôhoku Math. J. 13 (1961) 216-240 MR0147579

[10] T Bröcker, T tom Dieck, Representations of compact Lie groups, Graduate Texts in Mathematics 98, Springer, New York (1985) MR781344

[11] J J Duistermaat, J A C Kolk, Lie groups, Springer, Berlin (2000) MR1738431

[12] J M Gómez, A basis for the diagonally signed-symmetric polynomials, Electron. J. Combin. 20 (2013) MR3158275

[13] J M Gómez, A Pettet, J Souto, On the fundamental group of $\operatorname{Hom}\left(\mathbb{Z}^{k}, G\right)$, Math. Z. 271 (2012) 33-44 MR2917131

[14] A Hatcher, Algebraic topology, Cambridge Univ. Press (2002) MR1867354

[15] M Hochster, J A Eagon, Cohen-Macaulay rings, invariant theory, and the generic perfection of determinantal loci, Amer. J. Math. 93 (1971) 1020-1058 MR0302643

[16] J Hollender, RM Vogt, Modules of topological spaces, applications to homotopy limits and $E_{\infty}$ structures, Arch. Math. (Basel) 59 (1992) 115-129 MR1170635

[17] J A Lind, Diagram spaces, diagram spectra and spectra of units, Algebr. Geom. Topol. 13 (2013) 1857-1935 MR3073903

[18] J P May, The geometry of iterated loop spaces, Lecture Notes in Mathematics 271, Springer, Berlin (1972) MR0420610

[19] J P May, $E_{\infty}$ spaces, group completions, and permutative categories, from: "New developments in topology”, (G Segal, editor), London Math. Soc. Lecture Note Ser. 11, Cambridge Univ. Press (1974) 61-93 MR0339152

[20] J McCleary, A user's guide to spectral sequences, 2nd edition, Cambridge Studies in Advanced Mathematics 58, Cambridge Univ. Press (2001) MR1793722

[21] D McDuff, G Segal, Homology fibrations and the "group-completion" theorem, Invent. Math. 31 (1975/76) 279-284 MR0402733

[22] E Michael, Topologies on spaces of subsets, Trans. Amer. Math. Soc. 71 (1951) 152182 MR0042109

[23] A Pettet, J Souto, Commuting tuples in reductive groups and their maximal compact subgroups, Geom. Topol. 17 (2013) 2513-2593 MR3190294

[24] D Quillen, Projective modules over polynomial rings, Invent. Math. 36 (1976) 167-171 MR0427303

[25] G Segal, Categories and cohomology theories, Topol. 13 (1974) 293-312 MR0353298 
[26] J Souto, A remark on the homotopy equivalence of $\mathrm{SU}_{n}$ and $\mathrm{SL}_{n}(\mathbb{C})(2010)$ arXiv: 1008.0816

[27] F Vaccarino, The ring of multisymmetric functions, Ann. Inst. Fourier (Grenoble) 55 (2005) 717-731 MR2149400

Department of Mathematics, University of British Columbia

1984 Mathematics Road, Room 121, Vancouver BC V6T 1Z2, Canada

Departmento de Matemáticas, Universidad Nacional de Colombia

Calle 59A No 63 - 20, Bloque 43, Medellín, Colombia

adem@math.ubc.ca, jmgomez0@unal.edu.co

Received: 9 June $2014 \quad$ Revised: 10 July 2014 
